\title{
A closed-form universal trivariate pair-copula
}

Werner Hürlimann

Correspondence:

whurlimann@bluewin.ch

Feldstrasse 145, CH-8004 Zürich,

Switzerland

\begin{abstract}
Based on the trivariate pair-copula construction for the bivariate linear circular copula by Perlman and Wellner (Symmetry 3:574-99, 2011) and the Theorem of Carathéodory, which states that any valid correlation matrix is a finite convex combination of extreme correlation matrices, we generate a class of closed-form analytical 3-universal copulas. We derive explicit product and lifting copula formulas for the set of all extremal correlation matrices. Our analytical proof makes use of a novel set of conditional copula inequalities, which are of independent interest.
\end{abstract}

Keywords: Pair-copula; Trivariate universality; Linear circular copula; Elliptical copula; Extremal correlation; Product copula; Lifting copula; Conditional product copula inequalities

MSC 2010: 60E15; 62E15; 62H05; 62H2O

\section{Introduction}

In practice, the joint normal transform method for dependence modelling, which consists of transforming the margins to normal distributions, induce a dependence structure and transform back, is rather popular (e.g. Kurowicka and Cooke (2006), Section 4.2). Applied to a random vector $\left(X_{1}, X_{2}, \ldots, X_{n}\right)$ with invertible continuous marginal distributions $\left(F_{1}, F_{2}, \ldots, F_{n}\right)$ this method follows three steps:

1) Specify the rank correlation matrix $r=\left(r_{i j}\right), 1 \leq i, j \leq n$, of this random vector, i.e. $r_{i j}=$ $\operatorname{Cov}\left[F_{i}\left(X_{i}\right), F_{j}\left(X_{j}\right)\right], 1 \leq i, j \leq n$.

2) A random sample $\left(Y_{1}, Y_{2}, \ldots, Y_{n}\right)$ is drawn from a joint normal distribution with standard normal margins and the specified rank correlation matrix $r=\left(r_{i j}\right)$.

3) Let $\Phi(x)$ denote the standard normal distribution. The random sample $\left(X_{1}, X_{2}, \ldots, X_{n}\right)$ with margins $\left(F_{1}, F_{2}, \ldots, F_{n}\right)$ and the rank correlation matrix $r=\left(r_{i j}\right)$ is obtained from the inverse probability transform through $\left(X_{1}, X_{2}, \ldots, X_{n}\right)=$ $\left(F_{1}^{-1}\left(\Phi\left(Y_{1}\right)\right), F_{2}^{-1}\left(\Phi\left(Y_{2}\right)\right), \ldots, F_{n}^{-1}\left(\Phi\left(Y_{n}\right)\right)\right.$.

From a theoretical point of view this procedure suffers from several drawbacks. Concerning (1), it is known that every 3-dimensional valid correlation matrix can be realized as a rank correlation matrix, i.e. there exists a trivariate uniform distribution with this rank correlation structure, the so-called 3-universality property of copulas. This result is first stated in Joe (1997), Exercise 4.17, pp. 137-138. Kurowicka and Cooke (2006), Section 4.4.6, p.102, produce such trivariate copulas using the pair-copula construction for the so-called "elliptical copula" (see also Kurowicka and Cooke (2001)).

(C) 2014 Hürlimann; licensee Springer. This is an open access article distributed under the terms of the Creative Commons Attribution License (http://creativecommons.org/licenses/by/2.0), which permits unrestricted use, distribution, and reproduction in any medium, provided the original work is properly cited. 
Another recent proof is Devroye and Letac (2010). However, it is not yet known whether copulas are $n$-universal for $n \geq 6$ (see Letac (2010) for $n<6$ ). Therefore (1) is in general not necessarily consistent. The statement (2) causes similar difficulties. Indeed, the compatibility conditions under which a joint normal distribution with a specified rank correlation matrix exist, imply that for any $n \geq 3$ there are compatibility counterexamples (see Hürlimann (2012a), Theorem 3.1 and Corollary 4.2). Moreover, the probability of incompatibility increases with the dimension (e.g. Kurowicka and Cooke (2001)), Section 4, Table 1, Ghosh and Henderson (2002), and Ghosh (2004), Figure 3.1, p. 67). On the other hand, the 3-universality of the elliptical pair-copula construction does not extend to the fourvariate case (e.g. Kurowicka and Cooke (2006), Example 4.9). Therefore, to enable a rigorous use of any valid rank correlation matrix, there is a need for a more comprehensive understanding of the class of universal copulas, at least in small dimensions.

In the present paper we offer an analytical approach to the pair-copula construction for the bivariate linear circular copula derived by Perlman and Wellner (2011), whose density obviously coincides with the "elliptical copula" introduced by Kurowicka et al. (2000). To the best of our knowledge Section 5 presents for the first time simple closed-form analytical 3-universal copula formulas. The possible extension to the multivariate case is a challenging topic for future research (see Section 6). A more detailed account of the content follows.

Section 2 recalls the trivariate pair-copula construction. We explain how the combined use of extremal correlation matrices and bivariate elliptical copulas lead to extremal lifting copulas whose bivariate product copula margins induce sharp bounds in the concordance order. The latter include the set of all feasible rank correlation matrices. Advocating the Theorem of Carathéodory, which states that any valid correlation matrix can be written as a finite convex combination of extreme correlation matrices, the generation of 3-universal copulas follows. Section 3 summarizes the needed bivariate linear circular copula formulas. Section 4 presents the analytical closed-form product copula formulas, and Section 5 states our main result about analytical 3-universal copulas. Section 6 contains a simple two-dimensional algorithm for generating random vectors with 3-universal linear circular copula, and many references to potential applications are provided. Detailed proofs follow in the Appendices 1 and 2.

\section{Trivariate linear circular pair-copula construction}

In response to the difficulties encountered with the multivariate normal copula, the pair-copula construction of multivariate copulas has become more and more popular as can be seen from the recent review by Czado (2010). The first pair-copula construction is due to Joe (1996), see also Joe (1997), Section 4.5. His construction is given in terms of distribution functions, while Bedford and Cooke (2001) Bedford and Cooke (2002) expressed these constructions in terms of densities, and Kurowicka and Cooke (2006), Section 6.4, designed various sampling algorithms for them. A trivariate restatement of these equivalent representations will play a major role.

As observed by Devroye and Letac (2010), any valid rank correlation matrix can be written as a finite convex combination of extreme correlation matrices, also called extreme points (Theorem of Carathéodory (1911) and Steinitz (1914)). By linearity it suffices to restrict the attention to the pair-copula construction for extreme points. This 
way a finite algorithm that permits the construction of 3-universal copulas in terms of the linear circular copula can be designed. The proposed approach is then made fully analytical in Section 3 through the derivation of simple closed-form analytical 3universal copula formulas for the extreme points.

Recall the structure of the extreme points for the set of all positive semi-definite $3 \times 3$ correlation matrices. They have necessarily rank 1 or 2 and take the form (Ycart(1985), Corollary, p. 611):

$$
\rho=\rho(a, b, c)=\left(\rho_{i j}\right)=\left(\begin{array}{ccc}
1 & \cos c & \cos b \\
\cos c & 1 & \cos a \\
\cos b & \cos a & 1
\end{array}\right), \quad a+b+c \equiv 0 \bmod 2 \pi .
$$

One notes that there are four extreme points of rank one, namely

$$
(a, b, c) \in\{(0,0,0),(0, \pi, \pi),(\pi, 0, \pi),(\pi, \pi, 0)\}
$$

and the rank two extreme points are characterized by the condition

$$
(\sin a, \sin b, \sin c) \neq(0,0,0)
$$

The following immediate consequence is crucial.

\section{Lemma 2.1}

The absolute value of the partial correlation of a $3 \times 3$ extreme correlation matrix of the form (2.1) is always one. More precisely, one has

$$
\rho_{12 ; 3}=\frac{\rho_{12}-\rho_{13} \rho_{23}}{\sqrt{\left(1-\rho_{13}^{2}\right) \cdot\left(1-\rho_{23}^{2}\right)}}=-\operatorname{sgn}(\sin a \cdot \sin b)= \pm 1
$$

\section{Proof}

Since $a+b+c \equiv 0 \bmod 2 \pi$ we have from the cosine addition law that $\cos c=\cos (-(a+b)=$ $\cos (a+b)=\cos a \cdot \cos b-\sin a \cdot \sin b$. Inserted in the defining relation for the partial correlation coefficient we get $\rho_{12 ; 3}=-\frac{\sin a \cdot \sin b}{|\sin a \cdot \sin b|}$, which implies (2.4). $\diamond$

Now, in a first step, let us assume that all joint, marginal and conditional distributions are absolutely continuous, and that the corresponding densities exist. Let $\left(X_{1}, X_{2}\right.$, $\left.X_{3}\right)$ be a trivariate random vector from the Fréchet space $F\left(F_{13}, F_{23}\right)$ of all trivariate distributions with given bivariate margins $F_{13}\left(x_{1}, x_{3}\right), F_{23}\left(x_{2}, x_{3}\right)$. Denote the marginal distributions by $F_{1}\left(x_{1}\right), F_{2}\left(x_{2}\right), F_{3}\left(x_{3}\right)$, the conditional distributions obtained from $F_{13}, F_{23}$ by $F_{1 \mid 3}\left(x_{1} \mid x_{3}\right), F_{2 \mid 3}\left(x_{2} \mid x_{3}\right)$, and the marginal densities by $f_{1}\left(x_{1}\right), f_{2}\left(x_{2}\right), f_{3}\left(x_{3}\right)$. Let $C_{13}\left(u_{1}\right.$, $\left.u_{3}\right), C_{23}\left(u_{2}, u_{3}\right)$, respectively $C_{12 \mid 3}\left(u_{1}, u_{2}\right)$, be copulas associated to the random vectors $\left(X_{1}, X_{3}\right),\left(X_{2}, X_{3}\right)$, respectively to the conditional random vector $\left(X_{1}, X_{2} \mid X_{3}\right)$, which necessarily exist by the Theorem of Sklar (1959). The mixture of conditional distributions defined by (e.g. Joe (1997), equation (4.37))

$$
\begin{aligned}
& F_{123}\left(x_{1}, x_{2}, x_{3}\right)=\int_{-\infty}^{x_{3}} C_{12 \mid 3}\left(F_{1 \mid 3}\left(x_{1} \mid z_{3}\right), F_{2 \mid 3}\left(x_{2} \mid z_{3}\right)\right) d F_{3}\left(z_{3}\right) \\
& =\int_{-\infty}^{x_{3}} C_{12 \mid 3}\left(\frac{\partial C_{13}\left(F_{1}\left(x_{1}\right), F_{3}\left(z_{3}\right)\right)}{\partial u_{3}}, \frac{\partial C_{23}\left(F_{2}\left(x_{2}\right), F_{3}\left(z_{3}\right)\right)}{\partial u_{3}}\right) f_{3}\left(z_{3}\right) d z_{3},
\end{aligned}
$$


is a proper trivariate distribution in $F\left(F_{13}, F_{23}\right)$. In terms of densities, the joint trivariate density corresponding to (2.5), if it exists, reads (e.g. Czado (2010) Section 2)

$$
\begin{aligned}
& f_{123}\left(x_{1}, x_{2}, x_{3}\right) \\
& =c_{13}\left(F_{1}\left(x_{1}\right), F_{3}\left(x_{3}\right)\right) c_{23}\left(F_{2}\left(x_{2}\right), F_{3}\left(x_{3}\right)\right) c_{12 \mid 3}\left(F_{1 \mid 3}\left(x_{1} \mid x_{3}\right), F_{2 \mid 3}\left(x_{2} \mid x_{3}\right)\right) f_{1}\left(x_{1}\right) f_{2}\left(x_{2}\right) f_{3}\left(x_{3}\right),
\end{aligned}
$$

where $c_{13}\left(u_{1}, u_{3}\right), c_{23}\left(u_{2}, u_{3}\right), c_{12 \mid 3}\left(u_{1}, u_{2}\right)$ are the copula densities of $C_{13}\left(u_{1}, u_{3}\right), C_{23}\left(u_{2}, u_{3}\right)$, $C_{12 \mid 3}\left(u_{1}, u_{2}\right)$.

As we are looking for universal trivariate copulas we focus on uniform $[0,1]$ marginal distributions and copulas for bivariate marginal and bivariate conditional distributions. Let $\left(X_{1}, X_{2}, X_{3}\right)$ be a trivariate random vector with uniform [0,1] margins. A random sampling algorithm for the simulation of $\left(X_{1}, X_{2}, X_{3}\right)$ corresponding to (2.5) reads (e.g. Kurowicka and Cooke (2006), equation (6.2)):

$$
X_{3}=U_{3}, \quad X_{2}=F_{2 \mid 3}^{-1}\left(U_{2} \mid U_{3}\right), \quad X_{1}=F_{1 \mid 3}^{-1}\left(F_{12 \mid 3 ; U_{2}}^{-1}\left(U_{1}\right) \mid U_{3}\right)
$$

where $U_{1}, U_{2}, U_{3}$ are independent uniform $[0,1]$ random variables, $F_{1 \mid 3}^{-1}\left(x_{1} \mid x_{3}\right), F_{2 \mid 3}^{-1}$ $\left(x_{2} \mid x_{3}\right)$ are the inverse cumulative distributions of $F_{1 \mid 3}\left(x_{1} \mid x_{3}\right), F_{2 \mid 3}\left(x_{2} \mid x_{3}\right)$, and $F_{12 \mid 3 ; U_{2}}^{-1}$ $\left(x_{1}\right)$ denotes the inverse cumulative distribution for $X_{1}$ given $U_{2}$ under the conditional copula $C_{12 \mid 3}\left(u_{1}, u_{2}\right)$.

Kurowicka and Cooke (2006), Section 4.4.6, p.102, generate universal trivariate copulas with arbitrary valid rank correlation matrices using the sampling algorithm (2.7) based on the so-called "elliptical copula" for the involved copulas $C_{13}\left(u_{1}, u_{3}\right), C_{23}\left(u_{2}, u_{3}\right), C_{12 \mid 3}\left(u_{1}, u_{2}\right)$.

However, it seems that no attempt has been made so far to translate this Monte Carlo simulation procedure into closed-form analytical copula formulas. For this, it is appropriate to restate the representation (2.5) for the trivariate copula $C\left(u_{1}, u_{2}, u_{3}\right)$ associated to the uniform random vector $\left(X_{1}, X_{2}, X_{3}\right)$ as follows:

$$
C\left(u_{1}, u_{2}, u_{3}\right)=\int_{0}^{u_{3}} C_{12 \mid 3}\left(\frac{\partial}{\partial t} C_{13}\left(u_{1}, t\right), \frac{\partial}{\partial t} C_{23}\left(u_{2}, t\right)\right) d t
$$

It is important to remark that (2.8) is a special case of the following more general trivariate copula construction. Let $A(u, v), B(u, v)$ be two bivariate copulas, and let $C=\left\{C_{t}\right.$ $(u, v), t \in[0,1]\}$ be a family of bivariate copulas. Then, the mapping $A{ }^{*}{ }_{C} B:[0,1]^{3} \rightarrow$ $[0,1]$, defined by

$$
\left(A *_{C} B\right)\left(u_{1}, u_{2}, u_{3}\right)=\int_{0}^{u_{3}} C_{t}\left(\frac{\partial}{\partial t} A\left(u_{1}, t\right), \frac{\partial}{\partial t} B\left(u_{2}, t\right)\right) d t
$$

yields a trivariate copula, called the C-lifting of the copulas $A$ and $B$ (Durante et al. (2007a), Proposition 3.2). The bivariate copula margins of the trivariate copula (2.9) are $\left(A *{ }_{C} B\right)\left(u_{1}, u_{2}\right), A\left(u_{1}, u_{3}\right), B\left(u_{2}, u_{3}\right)$, where the bivariate copula

$$
\left(A *_{C} B\right)\left(u_{1}, u_{2}\right)=\int_{0}^{1} C_{t}\left(\frac{\partial}{\partial t} A\left(u_{1}, t\right), \frac{\partial}{\partial t} B\left(u_{2}, t\right)\right) d t
$$

is called the C-product of the copulas $A$ and $B$ (see Durante et al. (2007b) for details). Clearly, the special instance (2.8) is obtained for the (constant) conditional copula family $C_{t}(u, v) \equiv C_{12 \mid 3}(u, v), t \in[0,1]$. 
Specializing further to bivariate elliptical copulas and extremal correlation matrices with the property $\rho_{12 ; 3}= \pm 1$ (see Lemma 2.1), and finite convex combinations from these copulas, we claim that it suffices to consider the following two types of C-lifting copulas (2.8):

$$
\begin{aligned}
& C^{+}\left(u_{1}, u_{2}, u_{3}\right)=\int_{0}^{u_{3}} \min \left\{\frac{\partial}{\partial t} C_{13}\left(u_{1}, t\right), \frac{\partial}{\partial t} C_{23}\left(u_{2}, t\right)\right\} d t, \quad \text { if } \rho_{12 ; 3}=1, \\
& C^{-}\left(u_{1}, u_{2}, u_{3}\right)=\int_{0}^{u_{3}} \max \left\{\frac{\partial}{\partial t} C_{13}\left(u_{1}, t\right)+\frac{\partial}{\partial t} C_{23}\left(u_{2}, t\right)-1,0\right\} d t, \quad \text { if } \rho_{12 ; 3}=-1,
\end{aligned}
$$

where $C_{13}\left(u_{1}, u_{3}\right), C_{23}\left(u_{2}, u_{3}\right)$ are bivariate elliptical copulas with correlation parameters $r_{13}=\rho_{13}, r_{23}=\rho_{23}$. Indeed, choosing the Hoeffding-Fréchet bounds as conditional copulas $C_{12 \mid 3}\left(u_{1}, u_{2}\right)=M\left(u_{1}, u_{2}\right)=\min \left\{u_{1}, u_{2}\right\}$, resp. $C_{12 \mid 3}\left(u_{1}, u_{2}\right)=W\left(u_{1}, u_{2}\right)=\max \left\{u_{1}+\right.$ $\left.u_{2}-1,0\right\}$, corresponds to choosing the bivariate conditional elliptical copula for the extreme conditional correlations $r_{12 \mid 3}= \pm 1$ (note that the bivariate copula is a comprehensive family, which realizes any correlation value in the interval $(-1,1)$ ). But, for these elliptical copula choices, the conditional correlation is constant and coincides with the partial correlation (e.g. Kurowicka and Cooke (2006), Proposition 3.19, p.44). In particular, we have $r_{12 \mid 3}= \pm 1 \Leftrightarrow \rho_{12 ; 3}= \pm 1$, which shows that the construction (2.11) realizes the extremal correlation matrices with the property

$$
\rho_{12}=\rho_{13} \rho_{23} \pm \sqrt{\left(1-\rho_{13}^{2}\right) \cdot\left(1-\rho_{23}^{2}\right)}
$$

In particular, this means that the correlations of the product copulas $C^{+}\left(u_{1}, u_{2}\right)=$ $\left(C_{13} *{ }_{M} C_{23}\right)\left(u_{1}, u_{2}\right), C^{-}\left(u_{1}, u_{2}\right)=\left(C_{13} *{ }_{W} C_{23}\right)\left(u_{1}, u_{2}\right)$ coincide with (2.12). Moreover, according to Durante et al. (2007a), Corollary 4.1, we have for all bivariate copulas $C_{12}$ that are compatible with $C_{13}, C_{23}$ the sharp bounds in the concordance order:

$$
C^{-}\left(u_{1}, u_{2}\right) \leq C_{12}\left(u_{1}, u_{2}\right) \leq C^{+}\left(u_{1}, u_{2}\right)
$$

But $\rho_{13}, \rho_{23} \in[-1,1]$ are arbitrary and the correlation $\rho_{12}$ of $C_{12}$ in (2.12) varies between the two extreme bounds (2.12), hence all rank correlation matrices are feasible (see also the special case $n=3$ of Theorem 3.1 in Hürlimann (2012b)). The extreme lifting copulas (2.11) together with appropriate finite convex combinations generate a class of 3-universal copulas. In the next Sections, we show that the product and lifting copulas satisfy closed-form analytical expressions.

For reasons of symmetry it is more appropriate to work with uniform $[-1,1]$ random margins. Then a copula is defined on the centred cube $C_{n}=[-1,1]^{n}, n \geq 2$, with uniform $[-1,1]$ margins. In this setting and new notation, the lifting copulas (2.11) read:

$$
\begin{aligned}
& C^{+}(x, y, z)=\int_{-1}^{z} \min \left\{\frac{\partial}{\partial t} C_{13}(x, t), \frac{\partial}{\partial t} C_{23}(y, t)\right\} d t, \\
& C^{-}(x, y, z)=\int_{-1}^{z} \max \left\{\frac{\partial}{\partial t} C_{13}(x, t)+\frac{\partial}{\partial t} C_{23}(y, t)-\frac{1}{2}, 0\right\} d t, \quad(x, y, z) \in C_{3} .
\end{aligned}
$$


In a first step, we compute the bivariate product copulas with extremal correlations (2.12), i.e.

$$
\begin{aligned}
& C^{+}(x, y)=\int_{-1}^{1} \min \left\{\frac{\partial}{\partial t} C_{13}(x, t), \frac{\partial}{\partial t} C_{23}(y, t)\right\} d t, \\
& C^{-}(x, y)=\int_{-1}^{1} \max \left\{\frac{\partial}{\partial t} C_{13}(x, t)+\frac{\partial}{\partial t} C_{23}(y, t)-\frac{1}{2}, 0\right\} d t, \quad(x, y) \in C_{2} .
\end{aligned}
$$

For this we need the explicit formulas of the bivariate linear circular copula, which are summarized in the Section 3.

\section{The bivariate linear circular copula}

Starting point is the unique circular symmetric distribution on the unit disk $B_{2}$ in $R^{2}$ with uniform $[-1,1]$ margins. The associated so-called circular copula on $C_{2}$ is given by (Perlman and Wellner (2011), Theorem 3.1):

$$
C(u, v)=\frac{1}{4}(u+v+1)+\gamma_{0}(u, v), \quad \gamma_{0}(u, v)=\left\{\begin{array}{cc}
\alpha_{0}(u, v), & u^{2}+v^{2}<1, \\
\beta_{0}(u, v), & u^{2}+v^{2} \geq 1,
\end{array}\right.
$$

With

$$
\begin{aligned}
& \alpha_{0}(u, v)=\frac{1}{2 \pi} \cdot\left\{u \cdot \arcsin \left(\frac{v}{\sqrt{1-u^{2}}}\right)+v \cdot \arcsin \left(\frac{u}{\sqrt{1-v^{2}}}\right)-\arcsin \left(\frac{u v}{\sqrt{\left(1-u^{2}\right)\left(1-v^{2}\right)}}\right)\right\}, \\
& \beta_{0}(u, v)=\operatorname{sgn}(u v) \cdot \frac{1}{4}(|u|+|v|-1) .
\end{aligned}
$$

Taking partial derivatives one obtains the corresponding copula density

$$
c(u, v)=\frac{1}{2 \pi \sqrt{1-u^{2}-v^{2}}}, \quad(u, v) \in B_{2} .
$$

To obtain from this a one-parameter comprehensive family of copulas, let $(U, V) \sim$ $c(u, v)$ be the unique circular symmetric random vector on $B_{2}$. For each angle $\varphi \in\left(-\frac{\pi}{2}, \frac{\pi}{2}\right)$, set $r=\sin (\varphi) \in(-1,1)$, and consider the linear transformed random vector $\left(X, Y_{r}\right)$ defined by

$$
X=U, \quad Y_{r}=r \cdot U+\sqrt{1-r^{2}} \cdot V
$$

which by circular symmetry generates a copula on $C_{2}$. The support of $\left(X, Y_{r}\right)$ is the ellipse

$$
E_{r}=\left\{(x, y) \mid x^{2}+y^{2}-2 r x y<1-r^{2}\right\}
$$

Clearly, the correlation coefficient (that is equal to the rank correlation) of the random pair $\left(X, Y_{r}\right)$ is $r \in(-1,1)$. The corresponding comprehensive one-parameter family of linear circular copulas has the explicit representation (Perlman and Wellner (2011), Theorem 5.1):

$$
C_{r}(x, y)=\frac{1}{4}(x+y+1)+\gamma_{r}(x, y), \quad \gamma_{r}(x, y)=\left\{\begin{array}{cc}
\alpha_{r}(x, y), & (x, y) \in E_{r}, \\
\beta_{r}(x, y), & (x, y) \in C_{2} \backslash E_{r}
\end{array}\right.
$$


with

$$
\alpha_{r}(x, y)=\frac{1}{2 \pi} \cdot\left\{\begin{array}{l}
x \cdot \arcsin \left(\frac{y-r x}{\sqrt{\left(1-r^{2}\right)\left(1-x^{2}\right)}}\right)+y \cdot \arcsin \left(\frac{x-r y}{\sqrt{\left(1-r^{2}\right)\left(1-y^{2}\right)}}\right) \\
-\arcsin \left(\frac{x y-r}{\sqrt{\left(1-x^{2}\right)\left(1-y^{2}\right)}}\right)
\end{array}\right\},
$$

and

$$
\beta_{r}(x, y)=\left\{\begin{array}{lll}
\frac{1}{4}(x+y-1), & (x, y) \in L_{1}(r):=\left(C_{2} E_{r}\right) \cap\{x+y \geq 1+r\} \\
\frac{1}{4}(x-y+1), & (x, y) \in L_{2}(r):=\left(C_{2} E_{r}\right) \cap\{y-x \geq 1-r\} \\
\frac{1}{4}(-x-y-1), & (x, y) \in L_{3}(r):=\left(C_{2} E_{r}\right) \cap\{x+y \leq-1-r\} \\
\frac{1}{4}(y-x+1), & (x, y) \in L_{4}(r):=\left(C_{2} E_{r}\right) \cap\{y-x \leq-1+r\}
\end{array}\right.
$$

It is important to remark that the copula density coincides with the "elliptical copula" density introduced in Kurowicka et al. (2000) and extensively used in the book by Kurowicka and Cooke (2006). It is given by (Perlman and Wellner (2011), Proposition 5.1):

$$
c_{r}(x, y)=\frac{1}{2 \pi \sqrt{\left(1-r^{2}\right)\left(1-x^{2}\right)-(y-r x)^{2}}} \cdot 1_{E_{r}}(x, y), \quad(x, y) \in C_{2} .
$$

Since this family is obtained through linear transformation of the unique circular copula it is natural to use the name "linear circular copula". This new terminology clearly distinguishes it from the ubiquitous class of "elliptical copulas" that is generated by the multivariate elliptical distributions in any dimension. A further reason is that Perlman and Wellner (2011), Section 6, consider the possibility to generate non-linear circular copulas and provide an example.

For later use in Section 4, we also need expressions for the conditional linear circular copulas, i.e. the partial derivatives with respect to the arguments. For this, we first rewrite (3.6) for the outer part $C_{2} \backslash E_{r}$ of the ellipse as follows:

$$
C_{r}(x, y)=\left\{\begin{array}{l}
\frac{1}{2}(x+y), \quad(x, y) \in L_{1}(r) \\
\frac{1}{2}(x+1), \quad(x, y) \in L_{2}(r) \\
0, \quad(x, y) \in L_{3}(r) \\
\frac{1}{4}(y+1), \quad(x, y) \in L_{4}(r)
\end{array}\right.
$$

Through partial derivation we obtain the following formula

$$
\frac{\partial C_{r}(x, y)}{\partial y}= \begin{cases}\frac{1}{4}+\frac{1}{2 \pi} \arcsin \left(\frac{x-r y}{\sqrt{\left(1-r^{2}\right)\left(1-y^{2}\right)}}\right), & (x, y) \in E_{r} \\ \frac{1}{2}, & (x, y) \in L_{1}(r) \cup L_{4}(r) \\ 0, & (x, y) \in L_{2}(r) \cup L_{3}(r)\end{cases}
$$

\section{Analytical product copula formulas}

Using the explicit formulas (3.6)-(3.8) for the linear circular copula, it is now possible to derive simple analytical expressions for the extreme linear circular product copulas (2.15). Given are extreme correlation coefficients $\left(\rho_{13}, \rho_{23}, \rho_{12}^{ \pm}\right)$such that $\rho_{12}^{ \pm}=\rho_{13} \rho_{23} \pm$ $\sqrt{\left(1-\rho_{13}^{2}\right) \cdot\left(1-\rho_{23}^{2}\right)}, \rho_{13}, \rho_{23} \in[-1,1]$. Consider the linear circular copulas $C_{13}(x, y)=C_{r_{12}}$ $(x, y), C_{23}(x, y)=C_{r_{23}}(x, y)$ obtained from (3.6) by setting $r_{13}=\rho_{13}, \quad r_{23}=\rho_{23}$. The 
abbreviations $a=\sqrt{1-\rho_{13}^{2}}, \quad b=\sqrt{1-\rho_{23}^{2}}, c^{ \pm}=\rho_{23} a \mp b \rho_{13}$ are used throughout. Without loss of generality we assume that $a \geq b$.

\section{Proposition 4.1}

The upper extreme linear circular product copula $C^{+}(x, y)=\int_{-1}^{1} \min \left\{\frac{\partial}{\partial t} C_{13}(x, t), \frac{\partial}{\partial t} C_{23}(y, t)\right\} d t$, $(x, y) \in C_{2}$ satisfies the following analytical representation:

Case I: $c^{+}=r_{23} a-b r_{13} \geq 0$

(I.a) $C^{+}(x, y)=\frac{1}{2}(x+1), \quad a y-b x \geq c^{+}$,

(I.b) $C^{+}(x, y)=\frac{1}{2}(y+1)+C_{13}\left(x, \frac{a y-b x}{c^{+}}\right)-C_{23}\left(y, \frac{a y-b x}{c^{+}}\right), \quad-c^{+}<a y-b x<c^{+}$,

(I.c) $C^{+}(x, y)=\frac{1}{2}(y+1), \quad a y-b x \leq-c^{+}$.

Case II: $c^{+}=r_{23} a-b r_{13}<0$

$$
\begin{aligned}
& \text { (II.a) } C^{+}(x, y)=\frac{1}{2}(x+1), \quad a y-b x \geq-c^{+}, \\
& \text {(II.b) } C^{+}(x, y)=\frac{1}{2}(x+1)-C_{13}\left(x, \frac{a y-b x}{c^{+}}\right)+C_{23}\left(y, \frac{a y-b x}{c^{+}}\right), \quad c^{+}<a y-b x<-c^{+}, \\
& \text {(II.c) } C^{+}(x, y)=\frac{1}{2}(y+1), \quad a y-b x \leq c^{+} .
\end{aligned}
$$

The special case $c^{+}=0$ occurs exactly when $\rho_{13}=\rho_{23} \in[-1,1], \rho_{12}^{+}=1$, and (4.1) reduces to the formula $C^{+}(x, y)=\frac{1}{2}(x+1), \quad y \geq x, \quad C^{+}(x, y)=\frac{1}{2}(y+1), \quad y \leq x$.

\section{Proposition 4.2}

The lower extreme linear circular product copula $C^{-}(x, y)=\int_{-1}^{1} \max \left\{\frac{\partial}{\partial t} C_{13}(x, t)+\frac{\partial}{\partial t} C_{23}\right.$ $\left.(y, t)-\frac{1}{2}, 0\right\} d t,(x, y) \in C_{2}$, satisfies the following analytical representation:

Case I: $c^{-}=r_{23} a+b r_{13} \geq 0$

(I.a) $C^{-}(x, y)=\frac{1}{2}(x+y), \quad a y+b x \geq c^{-}$,

(I.b) $C^{-}(x, y)=C_{13}\left(x, \frac{a y+b x}{c^{-}}\right)+C_{23}\left(y, \frac{a y+b x}{c^{-}}\right)-\frac{1}{2}\left(1+\frac{a y+b x}{c^{-}}\right), \quad-c^{-}<a y+b x<c^{-}$,

(I.c) $C^{-}(x, y)=0, \quad a y+b x \leq-c^{-}$.

Case II: $c^{-}=r_{23} a+b r_{13}<0$

(II.a) $C^{-}(x, y)=\frac{1}{2}(x+y), \quad a y+b x \geq-c^{-}$

(II.b) $C^{-}(x, y)=\frac{1}{2}\left(x+y+1+\frac{a y+b x}{c^{-}}\right)-C_{13}\left(x, \frac{a y+b x}{c^{-}}\right)-C_{23}\left(y, \frac{a y+b x}{c^{-}}\right), \quad c^{-}<a y+b x<-c^{-}$,

(II.c) $C^{-}(x, y)=0, \quad a y+b x \leq c^{-}$.

The special case $c^{-}=0$ occurs exactly when $\rho_{23}=-\rho_{13} \in[-1,1], \rho_{12}^{-}=-1$, and (4.3) reduces to the formula $C^{-}(x, y)=\frac{1}{2}(x+y), x+y \geq 0, \quad C^{-}(x, y)=0, x+y \leq 0$. 


\section{Remark 4.1}

It has been stated after equation (2.12) that the (rank) correlations of the extreme linear circular product copulas coincide with $\rho_{12}^{ \pm}$. The obtained formulas (4.1)-(4.4) can be verified indirectly by showing that the rank correlations of these copulas are the right ones, i.e.

$$
\rho_{12}^{ \pm}=3 \cdot \int_{-1}^{1} \int_{-1}^{1} C^{ \pm}(x, y) d x d y-3
$$

This can be done through analytical calculation or numerical integral computation (the latter based on a computer algebra system, e.g. MATHCAD from MathSoft, Inc.). In fact, before conjectural statement and mathematical proof, we have first checked the simple case $c^{ \pm}=0$, for which $\rho_{12}^{ \pm}= \pm 1$, and have verified numerically the formulas (4.5) in the general case.

A direct analytical proof of the obtained formulas is based on a set of (firstly conjectured) conditional copula inequalities, which are of independent interest, and are proved in detail in Appendix 1 and 2. These inequalities are also of primordial importance in Section 5.

\section{Theorem 4.1}

(Upper conditional product copula or UPC inequalities) In the above notations the following inequalities hold for all $x, y, t \in[-1,1]$ :

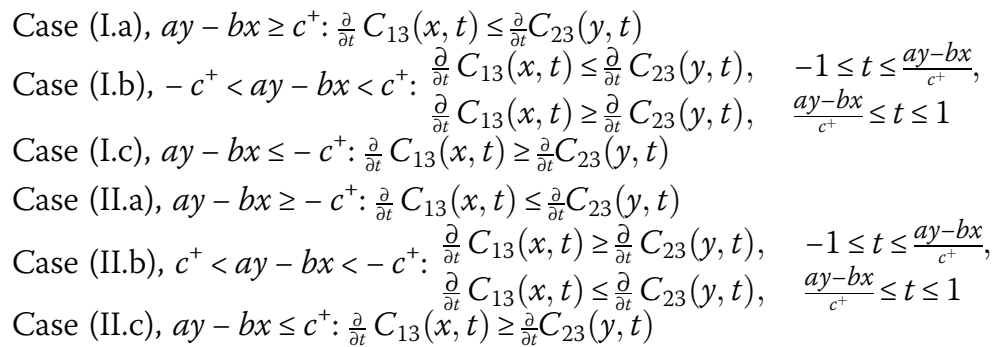

\section{Theorem 4.2}

(Lower conditional product copula or LPC inequalities) In the above notations the following inequalities hold for all $x, y, t \in[-1,1]$ :

$$
\begin{aligned}
& \text { Case (I.a), } a y+b x \geq c^{-}: \frac{\partial}{\partial t} C_{13}(x, t)+\frac{\partial}{\partial t} C_{23}(y, t)-\frac{1}{2} \geq 0 \\
& \text { Case (I.b), }-c^{-}<a y+b x<c^{-}: \begin{array}{l}
\frac{\partial}{\partial t} C_{13}(x, t)+\frac{\partial}{\partial t} C_{23}(y, t)-\frac{1}{2} \geq 0, \quad-1 \leq t \leq \frac{a y+b x}{c^{-}}, \\
\frac{\partial}{\partial t} C_{13}(x, t)+\frac{\partial}{\partial t} C_{23}(y, t)-\frac{1}{2} \leq 0, \quad \frac{a y+b x}{c^{-}} \leq t \leq 1
\end{array} \\
& \text { Case (I.c), } a y+b x \leq-c^{-}: \frac{\partial}{\partial t} C_{13}(x, t)+\frac{\partial}{\partial t} C_{23}(y, t)-\frac{1}{2} \leq 0 \\
& \text { Case (II.a), } a y+b x \geq-c^{-}: \frac{\partial}{\partial t} C_{13}(x, t)+\frac{\partial}{\partial t} C_{23}(y, t)-\frac{1}{2} \geq 0 \\
& \text { Case (II.b), } c^{-}<a y+b x<-c^{-}: \begin{array}{ll}
\frac{\partial}{\partial t} C_{13}(x, t)+\frac{\partial}{\partial t} C_{23}(y, t)-\frac{1}{2} \geq 0, & \frac{a y+b x}{c^{-}} \leq t \leq 1, \\
\frac{\partial}{\partial t} C_{13}(x, t)+\frac{\partial}{\partial t} C_{23}(y, t)-\frac{1}{2} \leq 0, & -1 \leq t \leq \frac{a y+b x}{c^{-}}
\end{array} \\
& \text {Case (II.c), } a y+b x \leq c^{-}: \frac{\partial}{\partial t} C_{13}(x, t)+\frac{\partial}{\partial t} C_{23}(y, t)-\frac{1}{2} \leq 0
\end{aligned}
$$

\section{Proof of the Propositions 4.1 and 4.2}

Inserting the inequalities from Theorem 4.1 (4.2) case by case into the upper (lower) product copula (2.15) shows the desired formulas. $\diamond$ 


\section{Analytical closed-form 3-universal copulas}

Based on the relevant conditional copula inequalities provided in the Theorems 4.1 and 4.2, we derive first analytical closed-form expressions for the extreme linear circular lifting copulas (2.14). We use the same notations, abbreviations and assumptions as in the preceding Section.

\section{Theorem 5.1}

The upper extreme linear circular lifting copula (2.14) satisfies the following analytical representation:

Case I: $c^{+}=r_{23} a-b r_{13} \geq 0$

(I.a) $C^{+}(x, y, z)=C_{13}(x, z), \quad a y-b x \geq c^{+}$,

(I.b) $C^{+}(x, y, z)=C_{13}\left(x, \frac{a y-b x}{c^{+}}\right)+C_{23}(y, z)-C_{23}\left(y, \frac{a y-b x}{c^{+}}\right), \quad z>\frac{a y-b x}{c^{+}}>-1$, $C^{+}(x, y, z)=C_{13}(x, z), \quad z \leq \frac{a y-b x}{c^{+}}<1$,

(I.c) $C^{+}(x, y)=C_{23}(y, z), \quad a y-b x \leq-c^{+}$.

Case II: $c^{+}=r_{23} a-b r_{13}<0$

$$
\begin{aligned}
\text { (II.a) } C^{+}(x, y, z) & =C_{13}(x, z), \quad a y-b x \geq-c^{+}, \\
\text {(II.b) } C^{+}(x, y, z) & =C_{13}(x, z)-C_{13}\left(x, \frac{a y-b x}{c^{+}}\right)+C_{23}\left(y, \frac{a y-b x}{c^{+}}\right), \quad z>\frac{a y-b x}{c^{+}}>-1, \\
C^{+}(x, y, z) & =C_{23}(y, z), \quad z \leq \frac{a y-b x}{c^{+}}<1, \\
\text { (II.c) } C^{+}(x, y, z) & =C_{23}(y, z), \quad a y-b x \leq c^{+} .
\end{aligned}
$$

The special case $c^{+}=0$ occurs exactly when $r=\rho_{13}=\rho_{23} \in[-1,1], \rho_{12}^{+}=1$, and (5.1) reduces to the formula $C^{+}(x, y, z)=C_{r}(x, z), y \geq x, C^{+}(x, y, z)=C_{r}(y, z), y \leq x$.

\section{Theorem 5.2}

The lower extreme linear circular lifting copula (2.14) satisfies the following analytical representation:

$$
\begin{aligned}
& \text { Case I: } c^{-}=r_{23} a+b r_{13} \geq 0 \\
& \begin{array}{l}
\text { (I.a) } C^{-}(x, y, z)=C_{13}(x, z)+C_{23}(y, z)-\frac{1}{2}(1+z), \quad a y+b x \geq c^{-}, \\
\text {(I.b) } C^{-}(x, y, z)=C_{13}\left(x, \min \left\{\frac{a y+b x}{c^{-}}, z\right\}\right)+C_{23}\left(y, \min \left\{\frac{a y+b x}{c^{-}}, z\right\}\right) \\
\quad-\frac{1}{2}\left(1+\min \left\{\frac{a y+b x}{c^{-}}, z\right\}\right), \quad-c^{-}<a y+b x<c^{-}, \\
\text {(I.c) } C^{-}(x, y, z)=0, \quad a y+b x \leq-c^{-} .
\end{array}
\end{aligned}
$$

Case II: $c^{-}=r_{23} a+b r_{13}<0$

$$
\begin{aligned}
& \text { (II.a) } C^{-}(x, y, z)=C_{13}(x, z)+C_{23}(y, z)-\frac{1}{2}(1+z), \quad a y+b x \geq-c^{-}, \\
& \text {(II.b) } C^{-}(x, y, z)=C_{13}(x, z)-C_{13}\left(x, \frac{a y+b x}{c^{-}}\right)+C_{23}(y, z)-C_{23}\left(y, \frac{a y+b x}{c^{-}}\right), \\
& z>\frac{a y+b x}{c^{-}}>-1, \quad C^{-}(x, y, z)=0, \quad z \leq \frac{a y+b x}{c^{-}}<1, \\
& \text { (II.c) } C^{-}(x, y, z)=0, \quad a y+b x \leq c^{-} .
\end{aligned}
$$

The special case $c^{-}=0$ occurs exactly when $\rho_{13}=\rho_{23}=0, \rho_{12}^{-}=-1$, and (5.3) reduces to the formula $C^{-}(x, y, z)=C_{0}(x, z)+C_{0}(y, z)-\frac{1}{2}(1+z), \quad x+y \geq 0, \quad C^{-}(x, y, z)=$ $0, x+y \leq 0$. 
Proof of the Theorems 5.1 and 5.2

Inserting the inequalities from Theorem 4.1 (4.2) case by case into the upper (lower) lifting copula (2.14) shows the desired formulas. $\diamond$

By the Theorem of Carathéodory, the obtained results show the existence of a 3universal linear circular based copula. However, instead of linearly combining four extreme points (Carathéodory's result), two such points suffice here, as shown in the next main result. Recall that the linear circular lifting copulas $C^{ \pm}(x, y, z)$ belong to the extreme points $\rho^{ \pm}=\left(\rho_{13}, \rho_{23}, \rho_{12}^{ \pm}\right)$.

\section{Theorem 5.3}

(3-universal linear circular copula) Let $\rho=\left(\rho_{13}, \rho_{23}, \rho_{12}\right)$ belong to an arbitrary $3 \times 3$ positive semi-definite rank correlation matrix. Then, the following 3-copula is universal:

$$
\begin{aligned}
& C_{\rho}(x, y, z)=\lambda^{-} C^{-}(x, y, z)+\lambda^{+} C^{+}(x, y, z), \quad(x, y, z) \in C_{3}, \\
& \lambda^{-}=\frac{1}{2} \frac{\rho_{12}^{+}-\rho_{12}}{\sqrt{\left(1-\rho_{13}^{2}\right) \cdot\left(1-\rho_{23}^{2}\right)}} \geq 0, \quad \lambda^{+}=\frac{1}{2} \frac{\rho_{12}-\rho_{12}^{-}}{\sqrt{\left(1-\rho_{13}^{2}\right) \cdot\left(1-\rho_{23}^{2}\right)}} \geq 0, \quad \lambda^{-}+\lambda^{+}=1 .
\end{aligned}
$$

\section{Proof}

This follows from the preceding results by noting that $\rho=\lambda^{-} \rho^{-}+\lambda^{+} \rho^{+} . \diamond$

\section{Applications and conclusions}

As an immediate statistical application, we show that the sampling algorithm for trivariate canonical vine copulas (Kurowicka and Cooke (2006), Section 6.4.2) reduces to a two-dimensional simulation algorithm for the uniform $[0,1]$ random vectors $X^{ \pm}=$ $\left(X_{1}^{ \pm}, X_{2}^{ \pm}, X_{3}^{ \pm}\right)$with lower and upper extreme linear circular lifting copulas $C^{ \pm}(u, v, w),(u$, $v, w) \in[0,1]^{3}$. Invoking Theorem 5.3 , this yields below a simple two-dimensional algorithm for generating random vectors $X=\left(X_{1}, X_{2}, X_{3}\right)$ with 3-universal linear circular copula. To show this, one needs some regression properties of the linear circular copula of Section 3.

\section{Proposition 6.1}

(Kurowicka and Cooke (2006), Proposition 3.18) Let $U, V$ be uniform $[0,1]$ random variables joined by the linear circular copula with correlation $\rho$.

Then one has

$$
\begin{aligned}
& E[V \mid U]=\frac{1}{2}(1-\rho)+\rho U \\
& \operatorname{Var}[V \mid U]=\frac{1}{2}\left(1-\rho^{2}\right) U(1-U) \\
& F_{V \mid U}(v \mid u)=\frac{1}{2}+\frac{1}{\pi} \arcsin \left(\frac{v-\rho u-\frac{1}{2}(1-\rho)}{\sqrt{\left(1-\rho^{2}\right) u(1-u)}}\right), \quad u, v \in[0,1] \\
& F_{V \mid U}^{-1}(\xi \mid u)=\frac{1}{2}(1-\rho)+\rho u+\sqrt{\left(1-\rho^{2}\right) u(1-u)} \cdot \sin \left(\pi \xi-\frac{\pi}{2}\right), \quad u, \xi \in[0,1]
\end{aligned}
$$

Of further relevance is the following result, which states that conditional correlations are constant and equal to partial correlations for the linear circular copula. 


\section{Proposition 6.2}

Kurowicka and Cooke (2006), Proposition 3.19) Let $U_{1}, U_{2}, U_{3}$ be uniform $[0,1]$ random variables, and let $U_{1}, U_{2}$ and $U_{2}, U_{3}$ be joined by the linear circular copula with correlations $\rho_{12}$ and $\rho_{13}$ respectively. Assume that the conditional copula for $U_{2}, U_{3}$ given $U_{1}$ does not depend on $U_{1}$. Then, the conditional correlation $\rho_{23 \mid 1}=\rho\left(U_{2}\left|U_{1}, U_{3}\right|\right.$ $\left.U_{1}\right)$ is constant in $U_{1}$ and $\rho_{23 \mid 1}=\rho_{23 ; 1}=\frac{\rho_{23}-\rho_{12} \rho_{13}}{\sqrt{\left(1-\rho_{12}^{2}\right) \cdot\left(1-\rho_{23}^{2}\right)}}$.

Now, let $U_{1}, U_{2}, U_{3}$ be uniform $[0,1]$ random variables. To generate a random vector $X=\left(X_{1}, X_{2}, X_{3}\right)$ with valid rank correlation matrix $\rho=\left(\rho_{13}, \rho_{23}, \rho_{12}\right)$, one uses the general sampling algorithm by Kurowicka and Cooke (2006), Section 6.4.2, which in the linear circular copula case reads as follows (use equation (6.4)):

$$
\begin{aligned}
& X_{1}=U_{1}, \quad X_{2}=F_{\rho_{12} ; U_{1}}^{-1}\left(U_{2}\right)=\frac{1}{2}\left(1-\rho_{12}\right)+\rho_{12} U_{1}+\sqrt{\left(1-\rho_{12}^{2}\right) U_{1}\left(1-U_{1}\right)} \cdot \sin \left(\pi U_{2}-\frac{\pi}{2}\right), \\
& X_{3}=F_{\rho_{13} ; U_{1}}^{-1}\left(F_{\rho_{23 \mid 1} ; U_{2}}^{-1}\left(U_{3}\right)\right)=\frac{1}{2}\left(1-\rho_{13}\right)+\rho_{13} U_{1}+\sqrt{\left(1-\rho_{13}^{2}\right) U_{1}\left(1-U_{1}\right)} \cdot \sin \left(\pi F_{\rho_{23 \mid} ; U_{2}}^{-1}\left(U_{3}\right)-\frac{\pi}{2}\right), \\
& F_{\rho_{23 \mid 1} ; U_{2}}^{-1}\left(U_{3}\right)=\frac{1}{2}\left(1-\rho_{23 \mid 1}\right)+\rho_{23 \mid 1} U_{2}+\sqrt{\left(1-\rho_{23 \mid 1}^{2}\right) U_{2}\left(1-U_{2}\right)} \cdot \sin \left(\pi U_{3}-\frac{\pi}{2}\right) .
\end{aligned}
$$

For the extreme points $\rho^{ \pm}=\left(\rho_{13}, \rho_{23}^{ \pm}, \rho_{12}\right)$ with $\rho_{23}^{ \pm}=\rho_{12} \rho_{13} \pm \sqrt{\left(1-\rho_{12}^{2}\right) \cdot\left(1-\rho_{13}^{2}\right)}$ one has $\rho_{23 \mid 1}^{ \pm}=\rho_{23 ; 1}^{ \pm}= \pm 1$ by Proposition 6.2 (see also Kurowicka and Cooke (2006), p.102). To generate uniform $[0,1]$ random vectors $X^{ \pm}=\left(X_{1}^{ \pm}, X_{2}^{ \pm}, X_{3}^{ \pm}\right)$with correlation matrices $\rho^{ \pm}$, one uses two times the preceding equations. In case $\rho_{23 \mid 1}^{+}=1$ one has $F_{\rho_{23 \mid 1}^{+} ; U_{2}}^{-1}\left(U_{3}\right)=U_{2}$, and in case $\rho_{23 \mid 1}^{-}=-1$ one has $F_{\rho_{23 \mid 1}^{-} ; U_{2}}^{-1}\left(U_{3}\right)=1-U_{2}$.

It follows that

$$
\begin{aligned}
& X_{1}^{ \pm}=U_{1}, \quad X_{2}^{ \pm}=\frac{1}{2}\left(1-\rho_{12}\right)+\rho_{12} U_{1}+\sqrt{\left(1-\rho_{12}^{2}\right) U_{1}\left(1-U_{1}\right)} \cdot \sin \left(\pi U_{2}-\frac{\pi}{2}\right) \\
& X_{3}^{ \pm}=\frac{1}{2}\left(1-\rho_{13}\right)+\rho_{13} U_{1} \pm \sqrt{\left(1-\rho_{13}^{2}\right) U_{1}\left(1-U_{1}\right)} \cdot \sin \left(\pi U_{2}-\frac{\pi}{2}\right)
\end{aligned}
$$

\section{Theorem 6.1}

Let $U_{1}, U_{2}$ be uniform $[0,1]$ random variables, and let $\rho=\left(\rho_{13}, \rho_{23}, \rho_{12}\right)$ be a valid rank correlation matrix. Then, the sampling procedure

$$
\begin{aligned}
& X_{1}=U_{1}, \quad X_{2}=\frac{1}{2}\left(1-\rho_{12}\right)+\rho_{12} U_{1}+\sqrt{\left(1-\rho_{12}^{2}\right) U_{1}\left(1-U_{1}\right)} \cdot \sin \left(\pi U_{2}-\frac{\pi}{2}\right) \\
& X_{3}=\frac{1}{2}\left(1-\rho_{13}\right)+\rho_{13} U_{1}+\rho_{23 ; 1} \sqrt{\left(1-\rho_{13}^{2}\right) U_{1}\left(1-U_{1}\right)} \cdot \sin \left(\pi U_{2}-\frac{\pi}{2}\right)
\end{aligned}
$$

generates a random vector with 3-universal linear circular copula that has correlation matrix $\rho$.

Proof

With a permutation of indices, one has by Theorem 5.3 that $X=\lambda^{-} X^{-}+\lambda^{+} X^{+}$, with $\lambda^{-}=\frac{1}{2} \frac{\rho_{23}^{+}-\rho_{23}}{\sqrt{\left(1-\rho_{12}^{2}\right) \cdot\left(1-\rho_{13}^{2}\right)}} \geq 0, \quad \lambda^{+}=\frac{1}{2} \frac{\rho_{23}-\rho_{23}^{-}}{\sqrt{\left(1-\rho_{12}^{2}\right) \cdot\left(1-\rho_{13}^{2}\right)}} \geq 0, \quad \lambda^{-}+\lambda^{+}=1$.

The result follows by combining the equations (6.5) noting that $\lambda^{+}-\lambda^{-}=\rho_{23 ; 1}$. $\diamond$

Let us conclude. Since simulation algorithms are almost always used in vine copula applications, any dimension reduction of such algorithms is a significant computational improvement. Vine copulas have received a lot of attention in recent years with applications in nearly all branches of mathematical sciences. Some further representative works on theory and applications of (vine) copulas include Cherubini et al. (2004), 
Mauguis and Guégan (2010), Vaz de Melo Mendes et al. (2010), Hürlimann (2011), Joe and Kurowicka (2011), Bernard and Czado (2012), Czado et al. (2012), Allen et al. (2013), Brechmann and Czado (2013), etc. Vine copulas are especially useful in higher dimensions. Unfortunately, due to its complexity, the present work could not be so far extended beyond dimension three. This is an important challenge for future research.

For $n<6$ Letac (2010) has announced some universal copulas, which use quite advanced mathematics, however. As stated by him, a difficulty lies in the lack of an easy characterization of the extreme points of the convex set of correlation matrices of order $n$. One can ask whether in higher dimensions the explicit recursive closed form correlation bounds for positive semi-definite correlation matrices derived in Hürlimann (2012b) are of help in this matter, as is the case in dimension three.

Explicit simpler constructions than ours are also open for investigation. Note that one of the most elementary approach, which is based on bivariate linear Spearman copulas, though completely classified, does not lead to 3-universal copulas, as shown in Hürlimann (2012c).

\section{Appendix 1}

\section{Some preliminary identities and inequalities}

We begin with some preliminary material that will be used throughout in Appendix 2 . Due to the structure of the bivariate linear circular copula, the analysis of the conjectured inequalities requires a case by case analysis of whether or not the coordinates $(x, t)$, respectively $(y, t)$, belong to one of the following disjoint regions of the centred square $C_{2}$. For $(x, t)$ these are the regions defined and denoted by

$$
\begin{aligned}
& E\left(r_{13}\right)=\left\{(x, t): x^{2}+t^{2}-2 r_{13} x t<1-r_{13}^{2}\right\}: \text { inner of ellipse } \\
& E^{-}\left(r_{13}\right)=\left\{(x, t): t \leq r_{13} x-\sqrt{\left(1-r_{13}^{2}\right)\left(1-x^{2}\right)}\right\}: \text { lower outer part of ellipse } \\
& E^{+}\left(r_{13}\right)=\left\{(x, t): t \geq r_{13} x+\sqrt{\left(1-r_{13}^{2}\right)\left(1-x^{2}\right)}\right\}: \text { upper outer part of ellipse } \\
& L_{1}\left(r_{13}\right)=\left(C_{2} \backslash E\left(r_{13}\right)\right) \cap\left\{x+t \geq 1+r_{13}\right\}: \text { outer part of ellipse above line in } 1^{\text {st }} \text { quadrant } \\
& L_{2}\left(r_{13}\right)=\left(C_{2} \backslash E\left(r_{13}\right)\right) \cap\left\{t-x \geq 1-r_{13}\right\}: \text { outer part of ellipse above line in } 2^{\text {nd }} \text { quadrant } \\
& L_{3}\left(r_{13}\right)=\left(C_{2} \backslash E\left(r_{13}\right)\right) \cap\left\{x+t \leq-1-r_{13}\right\}: \text { outer part of ellipse below line in } 3^{\text {rd }} \text { quadrant } \\
& L_{4}\left(r_{13}\right)=\left(C_{2} \backslash E\left(r_{13}\right)\right) \cap\left\{t-x \leq-1+r_{13}\right\}: \text { outer part of ellipse below line in } 4^{\text {th }} \text { quadrant }
\end{aligned}
$$

For $(y, t)$ these are the disjoint regions defined and denoted by

$$
\begin{aligned}
& E\left(r_{23}\right)=\left\{(y, t): y^{2}+t^{2}-2 r_{23} y t<1-r_{23}^{2}\right\}: \text { inner of ellipse } \\
& E^{-}\left(r_{23}\right)=\left\{(y, t): t \leq r_{23} y-\sqrt{\left(1-r_{23}^{2}\right)\left(1-y^{2}\right)}\right\}: \text { lower outer part of ellipse } \\
& E^{+}\left(r_{23}\right)=\left\{(y, t): t \geq r_{13} y+\sqrt{\left(1-r_{23}^{2}\right)\left(1-y^{2}\right)}\right\}: \text { upper outer part of ellipse } \\
& L_{1}\left(r_{23}\right)=\left(C_{2} \backslash E\left(r_{23}\right)\right) \cap\left\{y+t \geq 1+r_{23}\right\}: \text { outer part of ellipse above line in } 1^{\text {st }} \text { quadrant } \\
& L_{2}\left(r_{23}\right)=\left(C_{2} \backslash E\left(r_{23}\right)\right) \cap\left\{t-y \geq 1-r_{23}\right\}: \text { outer part of ellipse above line in } 2^{\text {nd }} \text { quadrant } \\
& L_{3}\left(r_{23}\right)=\left(C_{2} \backslash E\left(r_{23}\right)\right) \cap\left\{y+t \leq-1-r_{23}\right\}: \text { outer part of ellipse below line in } 3^{\text {rd }} \text { quadrant } \\
& L_{4}\left(r_{23}\right)=\left(C_{2} \backslash E\left(r_{23}\right)\right) \cap\left\{t-y \leq-1+r_{23}\right\}: \text { outer part of ellipse below line in } 4^{\text {th }} \text { quadrant }
\end{aligned}
$$

We note that involved expressions of the type $u v \pm \sqrt{\left(1-u^{2}\right)\left(1-v^{2}\right)}$ necessarily belong to the interval $[-1,1]$ in virtue of the following slightly more general property. 


\section{Lemma A1.1}

For $x, y, z \in[-1,1]$ the function defined by $w(x, y, z)=x y+z \sqrt{\left(1-x^{2}\right)\left(1-y^{2}\right)}$ takes values in $[-1,1]$.

\section{Proof}

See the proof of Lemma 4.7 in Kurowicka and Cooke (2006), pp. 121-122. $\diamond$

Some inequality properties of the function $w(x, y, \pm 1)=x y \pm \sqrt{\left(1-x^{2}\right)\left(1-y^{2}\right)}$ defined in Lemma A1.1 will be useful throughout the proofs.

\section{Lemma A1.2}

The following properties hold:

(P1) $w(x, y,-1) \leq x+y-1 \Leftrightarrow x+y \geq 0$

(P2) $w(x, y, 1) \geq x-y+1 \Leftrightarrow x-y \leq 0$

(P3) $\frac{\partial w(x, y,-1)}{\partial x} \geq 0 \Leftrightarrow x+y \geq 0, \quad \frac{\partial w(x, y,-1)}{\partial y} \geq 0 \quad \Leftrightarrow \quad x+y \geq 0$

(P4) $\frac{\partial w(x, y, 1)}{\partial x} \geq 0 \quad \Leftrightarrow \quad x-y \leq 0, \quad \frac{\partial w(x, y, 1)}{\partial y} \geq 0 \quad \Leftrightarrow \quad x-y \leq 0$

(P5) if $x, y<1$ then $w(x, y, 1)>x+y-1$

(P6) if $x, y<1$ then $w(x, y,-1)<1+y-x, w(x, y,-1)<1+x-y$

\section{Proof}

This is obtained without difficulty through standard algebraic calculation. $\diamond$

\section{Lemma A1.3}

The quantities $r_{13}, r_{23} \in[-1,1], \quad a=\sqrt{1-r_{13}^{2}} \geq b=\sqrt{1-r_{23}^{2}}$ and $c^{ \pm}=a r_{23} \mp b r_{13}$ satisfy the following identities:

$$
\frac{b \pm c^{ \pm} r_{13}}{a}=\frac{a-c^{ \pm} r_{23}}{b},\left(\frac{a-c^{ \pm} r_{23}}{b}\right)^{2}=1-\left(c^{ \pm}\right)^{2}
$$

\section{Proof}

The first identity is shown by verifying the following equivalences:

$$
\begin{aligned}
& b^{2} \pm b c^{ \pm} r_{13}=a^{2}-a c^{ \pm} r_{23} \Leftrightarrow c^{ \pm}\left(a r_{23} \pm b r_{13}\right)=a^{2}-b^{2} \\
& \Leftrightarrow \quad a^{2} r_{23}^{2}-b^{2} r_{13}^{2}=a^{2}-b^{2} \Leftrightarrow a^{2}\left(1-b^{2}\right)-b^{2}\left(1-a^{2}\right)=a^{2}-b^{2}
\end{aligned}
$$

The second one is shown similarly as follows:

$$
\begin{aligned}
& a^{2}-2 a c^{ \pm} r_{23}+\left(c^{ \pm}\right)^{2} r_{23}^{2}=b^{2}-b^{2}\left(c^{ \pm}\right)^{2} \quad \Leftrightarrow \quad a^{2}-2 a c^{ \pm} r_{23}+\left(c^{ \pm}\right)^{2}=b^{2} \\
& \Leftrightarrow \quad c^{ \pm}\left(2 a r_{23}-c^{ \pm}\right)=a^{2}-b^{2} \quad \Leftrightarrow \quad c^{ \pm}\left(a r_{23} \pm b \rho_{13}\right)=a^{2}-b^{2}
\end{aligned}
$$

Since the last identity is true by the preceding equivalences, the result is shown. $\diamond$

\section{Lemma A1.4}

The functions defined by

$$
u_{ \pm}=u_{ \pm}(x)=\frac{c^{ \pm} \cdot w\left(x, r_{13}, \pm 1\right) \pm b x}{a}, \quad v_{ \pm}=v_{ \pm}(y)= \pm\left(\frac{a y-c^{ \pm} \cdot w\left(y, r_{23}, \pm 1\right)}{b}\right)
$$


have the following inverse functions

$$
\begin{aligned}
& x=u_{ \pm}^{*}\left(u_{ \pm}\right)= \pm\left(\frac{a-c^{ \pm} r_{23}}{b}\right) \cdot u_{ \pm} \pm c^{ \pm} \cdot \sqrt{1-u_{ \pm}^{2}}=\left(\frac{c^{ \pm} r_{13} \pm b}{a}\right) \cdot u_{ \pm} \pm c^{ \pm} \cdot \sqrt{1-u_{ \pm}^{2}}, \\
& y=v_{ \pm}^{*}\left(v_{-}\right)= \pm\left(\frac{a-c^{ \pm} r_{23}}{b}\right) \cdot v_{ \pm} \pm c^{ \pm} \cdot \sqrt{1-v_{ \pm}^{2}}=\left(\frac{c^{ \pm} r_{13} \pm b}{a}\right) \cdot v_{ \pm} \pm c^{ \pm} \cdot \sqrt{1-v_{ \pm}^{2}}
\end{aligned}
$$

Proof

This is obtained through straightforward calculation using the identities (A1.1). $\diamond$

\section{Appendix 2}

Proof of the conditional product copula inequalities

Starting point are the following preliminary remarks. According to (3.11), in case ( $x$, $t) \in E\left(r_{13}\right), \quad(y, t) \in E\left(r_{23}\right)$, we have $\frac{\partial}{\partial t} C_{13}(x, t) \leq \frac{\partial}{\partial t} C_{23}(y, t)$ if, and only if

$$
\arcsin \left(\frac{x-r_{13} t}{a \sqrt{1-t^{2}}}\right) \leq \arcsin \left(\frac{y-r_{23} t}{b \sqrt{1-t^{2}}}\right) \Leftrightarrow c^{+} t \leq a y-b x
$$

and similarly, we have $\frac{\partial}{\partial t} C_{13}(x, t)+\frac{\partial}{\partial t} C_{23}(y, t)-\frac{1}{2} \geq 0$ if, and only if

$$
\arcsin \left(\frac{x-r_{13} t}{a \sqrt{1-t^{2}}}\right)+\arcsin \left(\frac{y-r_{23} t}{b \sqrt{1-t^{2}}}\right) \geq 0 \Leftrightarrow c^{-} t \leq a y+b x
$$

By symmetry it suffices to show the inequalities for Case I. The proof is done through exhaustive analysis of all possible disjoint regions that can contain the coordinates $(x, t)$, respectively $(y, t)$. We assume $c^{ \pm}>0$ and identify the cases $c^{ \pm}=0$ as limiting cases $c^{ \pm} \rightarrow 0$. Items related to Theorem 4.1 (4.2) are distinguished using the abbreviation UPC (LPC) for upper (lower) conditional product copula inequalities unless quantities involving $c^{ \pm}$ are used.

(1) $\quad(x, t) \in E^{-}\left(r_{13}\right)(y, t)$ arbitrary

(1.1) $x+r_{13} \geq 0$ (UPC), $x+r_{13}<0$ (LPC)

First of all, we note that $\frac{\partial}{\partial t} C_{13}(x, t)=\frac{1}{2}$ (UPC), $\frac{\partial}{\partial t} C_{13}(x, t)=0$ (LPC). To show this we use the properties of Lemma A1.2 and (3.11) as follows:

(UPC): $(\mathrm{P} 1) \Rightarrow t \leq w\left(x, r_{13},-1\right) \leq x+r_{13}-1 \Rightarrow(x, t) \in L_{4}\left(r_{13}\right)$

(LPC): (i) using (P6) of Lemma 3.2: $t \leq w\left(x, r_{13},-1\right)<1+r_{13}-x \Rightarrow(x, t) \notin L_{1}\left(r_{13}\right)$

(ii) using $x+r_{13}<0 t \geq-1>x+r_{13}-1 \Rightarrow(x, t) \notin L_{4}\left(r_{13}\right)$

Furthermore, we must have $y+r_{23} \geq 0$ in Cases (I.a) and (I.b), which is shown as follows:

(UPC) Case (I.a): if $y<-r_{23}$ then $0 \leq c^{+} \leq a y-b x<-a r_{23}-b r_{13}=-c^{+} \leq 0$ a contradiction

(UPC) Case (I.b): $y>\left(-c^{+}+b x\right) / a \geq-\left(c^{+}+b r_{13}\right) / a=-r_{23}$

(LPC) Case (I.a): if $y<-r_{23}$, then $0 \leq c^{-} \leq a y+b x<-a r_{23}-b r_{13}=-c^{-} \leq 0$ a contradiction

(LPC) Case (I.b): if $y<-r_{23}$ then $-c^{-}<a y+b x<-a r_{23}-b r_{13}=-c^{-}$a contradiction 
If one assumes that $t \leq w\left(y, r_{23},-1\right)$, then $\frac{\partial}{\partial t} C_{23}(y, t)=\frac{1}{2}$ in virtue of (3.11) because $(\mathrm{P} 1) \Rightarrow t \leq w\left(y, r_{23},-1\right) \leq y+r_{23}-1 \Rightarrow(y, t) \in L_{4}\left(r_{23}\right)$

We distinguish between three main cases:

(a) $c^{ \pm} t \geq a y \mp b x$ (Case (I.c) and possibly Case (I.b))

Since $\frac{\partial}{\partial t} C_{23}(y, t) \leq \frac{1}{2}$ we have necessarily $\frac{\partial}{\partial t} C_{13}(x, t)=\frac{1}{2} \geq \frac{\partial}{\partial t} C_{23}(y, t)$ (UPC), and $\frac{\partial}{\partial t} C_{13}$ $(x, t)+\frac{\partial}{\partial t} C_{23}(y, t)-\frac{1}{2} \leq 0$ (LPC), as in the desired inequalities.

(b) $c^{ \pm} t \leq a y \mp b x<c^{ \pm} \cdot w\left(y, r_{23},-1\right)$ (possible in Cases (I.a) and (I.b))

By the above we have $\frac{\partial}{\partial t} C_{13}(x, t)=\frac{\partial}{\partial t} C_{23}(y, t)=\frac{1}{2}(\mathrm{UPC})$ and $\frac{\partial}{\partial t} C_{13}(x, t)+\frac{\partial}{\partial t} C_{23}(y, t)-$ $\frac{1}{2}=0$ (LPC), which is consistent with the desired inequalities.

(c) $c^{ \pm} t \leq a y \mp b x$ and $c^{ \pm} \cdot w\left(y, r_{23},-1\right) \leq a y \mp b x$ (possible in Cases (I.a) and (I.b))

We derive the inequality (C1.1) $t \leq w\left(x, r_{13},-1\right) \leq w\left(y, r_{23},-1\right)$ which again implies that $\frac{\partial}{\partial t} C_{13}(x, t)=\frac{\partial}{\partial t} C_{23}(y, t)=\frac{1}{2}$ (UPC) and $\frac{\partial}{\partial t} C_{13}(x, t)+\frac{\partial}{\partial t} C_{23}(y, t)-\frac{1}{2}=0$ (LPC). We use the transformation of variable $z=z(y)= \pm\left(a y-c^{ \pm} \cdot w\left(y, r_{23},-1\right)\right) / b$ and its inverse $y=y(z)= \pm\left(a-c^{ \pm} r_{23}\right) / b \cdot z \pm c^{ \pm} \cdot \sqrt{1-z^{2}}$ in (A1.3), where the second " \pm " signs hold for both (UPC) and (LPC). We show (C1.1), that is $\Delta(x, y):=w\left(y, r_{23},-1\right)-w\left(x, r_{13},-1\right) \geq$ 0 under the constraints $-r_{13} \leq x \leq z(y)$ (UPC), $z(y) \leq x<-r_{13}$ (LPC), $-r_{23} \leq y \leq 1$. By (P3) of Lemma A1.2 the function $w\left(x, r_{13},-1\right)$ is increasing in $x$ (UPC), decreasing in $x$ (LPC). It follows that $\Delta(x, y) \geq \Delta^{*}(y, z(y)):=w\left(y, r_{23},-1\right)-w\left(z(y), r_{13},-1\right)$. Using the transformation $z(y)$ we can write $w\left(y, r_{23},-1\right)=(a y \mp b z(y)) / c^{ \pm}$. Making also use of the inverse $y=y(z)$ we see that (use the first identity in (A1.1)) $\Delta^{*}(z):=\Delta^{*}(y(z), z)=\frac{a y(z) \mp b z}{c^{ \pm}}-w$ $\left(z, r_{13},-1\right)=\frac{a}{c^{ \pm}}\left( \pm\left(a-c^{ \pm} r_{23}\right) / b \cdot z \pm c \cdot \sqrt{1-z^{2}}\right) \mp \frac{b}{c^{ \pm}} z-r_{13} z+a \sqrt{1-z^{2}}=a \sqrt{1-z^{2}}(1 \pm 1)$, which implies that $\Delta(x, y) \geq \Delta^{*}(y, z(y))=\Delta^{*}(z) \geq 0$ as desired.

(1.2) $x+r_{13}<0$ (UPC), $x+r_{13} \geq 0$ (LPC)

We have $\frac{\partial}{\partial t} C_{13}(x, t)=0 \quad \frac{\partial}{\partial t} C_{13}(x, t)=\frac{1}{2}$ (UPC), (LPC), which is shown as follows:

(UPC): (i) using (P6) of Lemma A1.2: $t \leq w\left(x, r_{13},-1\right)<1+r_{13}-x \Rightarrow(x, t) \notin L_{1}\left(r_{13}\right)$

(ii) using $x+r_{13}<0$ : $t \geq-1>x+r_{13}-1 \Rightarrow(x, t) \notin L_{4}\left(r_{13}\right)$

(LPC): (P1) of Lemma A1.2 $\Rightarrow t \leq w\left(x, r_{13},-1\right) \leq x+r_{13}-1 \Rightarrow(x, t) \in L_{4}\left(r_{13}\right)$

We distinguish between two main cases:

(a) $c^{ \pm} t \leq a y \mp b x$ (Case (I.a) and possibly Case (I.b))

We have $\frac{\partial}{\partial t} C_{13}(x, t)=0 \leq \frac{\partial}{\partial t} C_{23}(y, t)$ (UPC), $\frac{\partial}{\partial t} C_{13}(x, t)+\frac{\partial}{\partial t} C_{23}(y, t)-\frac{1}{2} \geq 0$ (LPC) as desired (b) $a y \mp b x \leq c^{ \pm} t \leq c^{ \pm} \cdot w\left(x, r_{13},-1\right)$ (Case (I.c) and possibly Case (I.b))

We show $\frac{\partial}{\partial t} C_{23}(y, t)=0$, which is consistent with the desired results $\frac{\partial}{\partial t} C_{13}(x, t) \geq \frac{\partial}{\partial t} C_{23}$ $(y, t)$ (UPC) and $\frac{\partial}{\partial t} C_{13}(x, t)+\frac{\partial}{\partial t} C_{23}(y, t)-\frac{1}{2} \leq 0$ (LPC). It suffices to show the following inequalities:

Case 1: $x \geq-\left(b+c^{+} r_{13}\right) / a$ (UPC), $x \leq\left(b-c^{-} r_{13}\right) / a(\mathrm{LPC})$

(C1.2.1) $t \leq w\left(x, r_{13},-1\right) \leq w\left(y, r_{23},-1\right)$, and $y+r_{23} \leq 0$

Case 2: $x \leq-\left(b+c^{+} r_{13}\right) / a(\mathrm{UPC}), x \geq\left(b-c^{-} r_{13}\right) / a(\mathrm{LPC})$

$(\mathrm{C} 1.2 .2) c^{ \pm} \cdot w\left(y, r_{23}, 1\right) \leq a y \mp b x \leq c^{ \pm} t \leq c^{ \pm} \cdot w\left(x, r_{13},-1\right)$, and $y-r_{23} \leq 0$ 
Indeed, if these conditions hold, then $\frac{\partial}{\partial t} C_{23}(y, t)=0$ in virtue of (3.11) as follows:

Case 1: (i) using (P6) of Lemma A1.2: $t \leq w\left(y, r_{23},-1\right)<1+r_{23}-y \Rightarrow(y, t) \notin L_{1}\left(r_{23}\right)$

(ii) using $y+r_{23} \leq 0$ : $t \geq-1 \geq y+r_{23}-1 \Rightarrow(y, t) \notin L_{4}\left(r_{23}\right)$

Case 2: (i) using $y-r_{23} \leq 0$ : $t \leq 1 \leq 1+r_{23}-y \Rightarrow(y, t) \notin L_{1}\left(r_{23}\right)$

(ii) using (P5) of Lemma A1.2: $t \geq w\left(y, r_{23}, 1\right)>y+r_{23}-1 \Rightarrow(y, t) \notin L_{4}\left(r_{23}\right)$

To show the above inequalities we use the transformation $z=z(x)=\left(c^{ \pm} \cdot w\left(x, r_{13},-1\right) \pm\right.$ $b x) / a$.

Case 1: The function $z(x)$ is increasing in the interval $-\left(b+c^{ \pm} r_{13}\right) / a \leq x<-r_{13}$ (UPC), respectively $z(x)$ is decreasing in the interval $-r_{13} \leq x \leq\left(b-c^{-} r_{13}\right) / a$ (LPC):

$$
\begin{aligned}
& z^{\prime}(x)=\left(c^{ \pm} r_{13} \pm b\right) / a+c^{ \pm} \cdot x / \sqrt{1-x^{2}} \geq 0 \quad \Leftrightarrow \quad x / \sqrt{1-x^{2}} \geq \alpha:=-\left( \pm b+c^{ \pm} r_{13}\right) / a c^{ \pm} \\
& \Leftrightarrow \quad x \geq \alpha / \sqrt{1+\alpha^{2}}=-\left( \pm b+c^{ \pm} r_{13}\right) / a
\end{aligned}
$$

In particular, by the restriction defining case (b), we have necessarily $y \leq z(x) \leq z\left(-r_{13}\right)=$ $\left(-c^{ \pm} \mp b r_{13}\right) / a=-r_{23} \Rightarrow y+r_{23} \leq 0$.

Now, we show that $\Delta(x, y)=w\left(y, r_{23},-1\right)-w\left(x, r_{13},-1\right) \geq 0$ under the constraints, $1 \leq y \leq z(x), \quad-\left(b+c^{+} r_{13}\right) / a \leq x<-r_{13}$ (UPC), $-r_{13} \leq x \leq\left(b-c^{-} r_{13}\right) / a$ (LPC). Since $w(y$, $\left.r_{23},-1\right)$ is decreasing in $y$ by (P3) of Lemma A1.2, we have $\Delta(x, y) \geq \Delta^{*}(x, z(x)):=w(z(x)$, $\left.r_{23},-1\right)-w\left(x, r_{13},-1\right)=w\left(z(x), r_{23},-1\right)-(a z(x) \mp b x) / c^{ \pm}$.

Since $z(x)$ is increasing and $z\left(-r_{13}\right)=-r_{23}, z\left(-\left(b+c^{+} r_{13}\right) / a\right)=-1$ (UPC), respectively $z(x)$ is decreasing and $z\left(-r_{13}\right)=-r_{23}, z\left(\left(b-c^{-} r_{13}\right) / a\right)=-1$, its inverse in (A1.3) takes the "+" sign (UPC) respectively the "-" sign (LPC), that is $x=x(z)= \pm\left(b \pm c^{ \pm} r_{13}\right) / a \cdot z \pm$ $c^{ \pm} \cdot \sqrt{1-z^{2}}$. Insert it into the function $\Delta^{*}(z)=\Delta^{*}(x(z), z)=w\left(z, r_{23},-1\right)-(a z \mp b x(z)) / c^{ \pm}$to see that $\Delta(x, y) \geq \Delta^{*}(x, z(x))=\Delta^{*}(z)=0$ as desired.

Case 2: Similarly to Case 1 , one shows that $z(x)$ is decreasing in the interval $-1 \leq x \leq-$ $\left(b+c^{+} r_{13}\right) / a$ (UPC), respectively $z(x)$ is increasing in the interval $\left(b-c^{-} r_{13}\right) / a \leq x \leq 1$ (LPC), and in particular $y \leq z(x) \leq z(-1)=-\left( \pm c^{ \pm} r_{13}+b\right) / a \leq r_{23}$, where the last inequality is true because $a r_{23}+b+c^{+} r_{13}=\left(b+c^{+}\right) \cdot\left(1+r_{13}\right) \geq 0$ (UPC), $a r_{23}+b-c^{-} r_{13} \geq c^{-} \cdot\left(1-r_{13}\right) \geq$ 0 (LPC).

Now, we show that $\Delta(x, y)=(a y \mp b x) / c^{ \pm}-w\left(y, r_{23}, 1\right) \geq 0$ under the constraints $-1 \leq$ $y \leq z(x),-1 \leq x \leq-\left(b+c^{+} r_{13}\right) / a$ (UPC), $\left(b-c^{-} r_{13}\right) / a \leq x \leq 1$ (LPC). The function $h(y)= \pm$ $\left(a y / c^{ \pm}-w\left(y, r_{23}, 1\right)\right)$ is decreasing (UPC), respectively increasing (LPC), in the interval $1 \leq y \leq-\left(b \pm c^{ \pm} r_{13}\right) / a$ :

$$
\begin{aligned}
& h^{\prime}(y)= \pm\left(a-c^{ \pm} r_{23}\right) / c^{ \pm} \pm b \cdot y / \sqrt{1-y^{2}} \leq(\geq) 0 \Leftrightarrow y / \sqrt{1-y^{2}} \leq \beta:=\left(c^{ \pm} r_{23}-a\right) / b c^{ \pm} \\
& \Leftrightarrow \quad y \leq \beta / \sqrt{1+\beta^{2}}=\left(c^{ \pm} r_{23}-a\right) / b=\mp\left(b \pm c^{ \pm} r_{13}\right) / a
\end{aligned}
$$

It follows that $\Delta(x, y) \geq \Delta^{*}(x, z(x))=(a z(x) \mp b x) / c^{ \pm}-w\left(z(x), r_{23}, 1\right)$. Since $z(x)$ is decreasing (UPC), respectively increasing (LPC), and $z\left(\mp\left(b \pm c^{ \pm} r_{13}\right) / a\right)=-1, z(\mp 1)=\mp\left(b \pm c^{ \pm} r_{13}\right) /$ $a$, its inverse in (A1.3) takes the "-" sign (UPC) respectively the "+" sign (LPC), that is we have $x=x(z)= \pm\left(b \pm c^{ \pm} r_{13}\right) / a \cdot z \mp c^{ \pm} \cdot \sqrt{1-z^{2}}$. Insert this expression into the function $\Delta^{*}(z)=\Delta^{*}(x(z), z)=(a z \mp b x(z)) / c^{ \pm}-w\left(z, r_{23}, 1\right)$ to see that $\Delta(x, y) \geq \Delta^{*}(x, z(x))=\Delta^{*}(z)=0$.

(2) $(y, t) \in E^{-}\left(r_{23}\right)(x, t)$ arbitrary

(2.1) $y+r_{23}<0$ 
We have $\frac{\partial}{\partial t} C_{23}(y, t)=0$, which follows from Lemma A1.2 and (3.11) as follows:

(i) using (P6) of Lemma A1.2: $t \leq w\left(y, r_{23},-1\right)<1+r_{23}-y \Rightarrow(y, t) \notin L_{1}\left(r_{23}\right)$

(ii) using $y+r_{23}<0$ : $t \geq-1>y+r_{23}-1 \Rightarrow(y, t) \notin L_{4}\left(r_{23}\right)$

Furthermore, we must have $x+r_{13}<0$ (UPC), respectively $x+r_{13} \geq 0$ (LPC), in Cases (I.a) and (I.b), which is shown as follows:

(UPC) Case (I.a): $a y-b x \geq c^{+} \Rightarrow x \leq\left(a y-c^{+}\right) / b<-\left(c^{+}+a r_{13}\right) / b \leq-r_{13}$, where the last inequality follows from $a r_{23}-b r_{13}+c^{+}=2 c^{+} \geq 0$

(UPC) Case (I.b): $a y-b x>-c^{+} \Rightarrow x<\left(c^{+}+a y\right) / b<\left(c^{+}-a r_{23}\right) / b=-r_{13}$

(LPC) Case (I.a): if $x<-r_{13}$, then $0 \leq c^{-} \leq a y+b x<-a r_{23}-b r_{13}=-c^{-} \leq 0$, a contradiction

(LPC) Case (I.b): if $x<-r_{13}$, then $-c^{-}<a y+b x<-a r_{23}-b r_{13}=-c^{-}$, a contradiction

In these Cases, if $t \leq w\left(x, r_{13},-1\right)$, then $\frac{\partial}{\partial t} C_{13}(x, t)=0$ (UPC), $\frac{\partial}{\partial t} C_{13}(x, t)=\frac{1}{2}(\mathrm{LPC})$ :

(UPC): (i) using (P6) of Lemma 3.2: $t \leq w\left(x, r_{13},-1\right)<1+r_{13}-x \Rightarrow(x, t) \notin L_{1}\left(r_{13}\right)$

(ii) using $x+r_{13}<0$ : $t \geq-1>x+r_{13}-1 \Rightarrow(x, t) \notin L_{4}\left(r_{13}\right)$

(LPC): (P1) of Lemma A1.2 $\Rightarrow x+r_{13} \geq 0 \Rightarrow w\left(x, r_{13},-1\right) \leq x+r_{13}-1 \Rightarrow(x, t) \in L_{4}\left(r_{13}\right)$ We have three main cases:

(a) $c^{ \pm} t \geq a y \mp b x$ (Case (I.c) and possibly Case (I.b))

We have necessarily $\frac{\partial}{\partial t} C_{13}(x, t) \geq \frac{\partial}{\partial t} C_{23}(y, t)=0 \quad(\mathrm{UPC}), \quad \frac{\partial}{\partial t} C_{13}(x, t)+\frac{\partial}{\partial t} C_{23}(y, t)-\frac{1}{2} \leq 0$ (LPC)

(b) $c^{ \pm} t \leq a y \mp b x<c^{ \pm} \cdot w\left(x, r_{13},-1\right)$ (possible in Cases (I.a) and (I.b))

By the above we have $\frac{\partial}{\partial t} C_{13}(x, t)=\frac{\partial}{\partial t} C_{23}(y, t)=0$ (UPC), $\frac{\partial}{\partial t} C_{13}(x, t)+\frac{\partial}{\partial t} C_{23}(y, t)-\frac{1}{2}=0$

(LPC), which is consistent with the desired inequalities.

(c) $c^{ \pm} t \leq a y \mp b x$ and $c^{ \pm} \cdot w\left(y, r_{23},-1\right) \leq a y \mp b x$ (possible in Cases (I.a) and (I.b))

We derive the inequality $(\mathrm{C} 2.1) t \leq w\left(y, r_{23},-1\right) \leq w\left(x, r_{13},-1\right)$, which again implies $\frac{\partial}{\partial t}$ $C_{13}(x, t)=\frac{\partial}{\partial t} C_{23}(y, t)=0 \quad$ (UPC), $\quad \frac{\partial}{\partial t} C_{13}(x, t)+\frac{\partial}{\partial t} C_{23}(y, t)-\frac{1}{2}=0 \quad$ (LPC). We use the transformation of variable $z=z(x)=\left(c^{ \pm} \cdot w\left(x, r_{13},-1\right) \pm b x\right) / a$ and its inverse $x=x(z)= \pm$ $\left(a-c^{ \pm} r_{23}\right) / b \cdot z \pm c^{ \pm} \cdot \sqrt{1-z^{2}}$ in (A1.3), where the second " \pm " signs hold for both (UPC) and (LPC). We show that $\Delta(x, y):=w\left(x, r_{13},-1\right)-w\left(y, r_{23},-1\right) \geq 0$ under the constraints $z$ $(x) \leq y<-r_{23}, \quad-1 \leq x<-r_{13}$ (UPC), $-r_{13} \leq x \leq 1$ (LPC). By (P3) of Lemma A1.2 the function $w\left(y, r_{23},-1\right)$ is decreasing in $y$. It follows that $\Delta(x, y) \geq \Delta^{*}(x, z(x))=w\left(x, r_{13},-1\right)-w$ $\left(z(x), r_{23},-1\right)=(a z(x) \mp b x) / c^{ \pm}-w\left(z(x), r_{23},-1\right)$.

Now, insert the inverse $x=x(z)$ into $\Delta^{* *}(z)=\Delta^{*}(x(z), z)=(a z \mp b x(z)) / c^{ \pm}-w\left(z, r_{23},-1\right)$ to see that $\Delta(x, y) \geq \Delta^{*}(x, z(x))=\Delta^{*}(z)=b \sqrt{1-z^{2}}(1 \pm 1) \geq 0$ as desired.

(2.2) $y+r_{23} \geq 0$

Property (P1) implies $t \leq w\left(y, r_{23},-1\right) \leq y+r_{23}-1 \Rightarrow(y, t) \in L_{4}\left(r_{23}\right) \Rightarrow \frac{\partial}{\partial t} C_{23}(y, t)=\frac{1}{2}$

We distinguish between two main cases:

(a) $c^{ \pm} t \leq a y \mp b x$ (Case (I.a) and possibly Case (I.b)) 
We have necessarily $\frac{\partial}{\partial t} C_{13}(x, t) \leq \frac{\partial}{\partial t} C_{23}(y, t)=\frac{1}{2} \quad(\mathrm{UPC}), \quad \frac{\partial}{\partial t} C_{13}(x, t)+\frac{\partial}{\partial t} C_{23}(y, t)-\frac{1}{2} \geq 0$ (LPC).

(b) $a y \mp b x \leq c^{ \pm} t \leq c^{ \pm} \cdot w\left(x, r_{13},-1\right)$ (Case (I.c) and possibly Case (I.b))

We show that $\frac{\partial}{\partial t} C_{13}(x, t)=\frac{1}{2}$ (UPC), $\frac{\partial}{\partial t} C_{13}(x, t)=0$ (LPC), which is consistent with $\frac{\partial}{\partial t}$ $C_{13}(x, t) \geq \frac{\partial}{\partial t} C_{23}(y, t)$ (UPC), $\frac{\partial}{\partial t} C_{13}(x, t)+\frac{\partial}{\partial t} C_{23}(y, t)-\frac{1}{2} \leq 0$ (LPC). It suffices to show the following inequalities:

Case 1: $y \leq\left(a-c^{ \pm} r_{23}\right) / b$

(C2.2.1) $t \leq w\left(y, r_{23},-1\right) \leq w\left(x, r_{13},-1\right.$, and $x+r_{13} \geq 0$ (UPC), $x+r_{13} \leq 0$ (LPC)

Case 2: $y \geq\left(a-c^{ \pm} r_{23}\right) / b$

(C2.2.2) $w\left(x, r_{13}, 1\right) \leq(a y-b x) / c \leq t \leq w\left(y, r_{23},-1\right)$, and $x-r_{13} \geq 0$ (UPC), $x-r_{13} \leq 0$ (LPC)

Indeed, if these conditions hold, then $\frac{\partial}{\partial t} C_{13}(x, t)=\frac{1}{2}$ (UPC), $\frac{\partial}{\partial t} C_{13}(x, t)=0$ (LPC) as follows:

(UPC) Case 1: Property (P1) implies $t \leq w\left(x, r_{13},-1\right) \leq x+r_{13}-1 \Rightarrow(x, t) \in L_{4}\left(r_{13}\right)$

(LPC) Case 1:

(i) using (P6) of Lemma 3.2: $t \leq w\left(x, r_{13},-1\right)<1+r_{13}-x \Rightarrow(x, t) \notin L_{1}\left(r_{13}\right)$

(ii) using $x+r_{13} \leq 0$ : $t \geq-1 \geq x+r_{13}-1 \Rightarrow(x, t) \notin L_{4}\left(r_{13}\right)$

(UPC) Case 2: Property (P2) implies $t \geq w\left(x, r_{13}, 1\right) \geq 1+r_{13}-x \Rightarrow(x, t) \in L_{1}\left(r_{13}\right)$

(LPC) Case 2:

(i) using $x-r_{13} \leq 0$ : $t \leq 1 \leq 1+r_{13}-x \Rightarrow(x, t) \notin L_{1}\left(r_{13}\right)$

(ii) using (P5) of Lemma 3.2: $t \geq w\left(x, r_{13}, 1\right)>x+r_{13}-1 \Rightarrow(x, t) \notin L_{4}\left(r_{13}\right)$

To show the above inequalities we use the transformation $z=z(y)= \pm\left(a y-c^{ \pm} \cdot w(y\right.$, $\left.\left.r_{23},-1\right)\right) / b$.

Case 1: The function $z(y)$ is increasing (UPC) (respectively decreasing (LPC)) in the interval $\quad-r_{23} \leq y \leq\left(a-c^{ \pm} r_{23}\right) / b: \quad z^{\prime}(y)= \pm\left(a-c^{ \pm} r_{23}\right) / b \mp c^{ \pm} \cdot y / \sqrt{1-y^{2}} \geq(\leq) 0 \quad \Leftrightarrow \quad y \leq$ $\left(a-c^{ \pm} r_{23}\right) / b$, hence $x \geq z(y) \geq z\left(-r_{23}\right)=\left(c^{+}-a r_{23}\right) / b=-r_{13}$ (UPC), $x \leq z(y) \leq z\left(-r_{23}\right)=$ $\left(a r_{23}-c^{-}\right) / b=-r_{13}$ (LPC). Now, we show that $\Delta(x, y)=w\left(x, r_{13},-1\right)-w\left(y, r_{23},-1\right) \geq 0$ under the constraints $z(y) \leq x \leq 1$ (UPC), $-1 \leq x \leq z(y)$ (LPC), $-r_{23} \leq y \leq\left(a-c^{ \pm} r_{23}\right) / b$.

Since $w\left(x, r_{13},-1\right)$ is increasing in $x$ (UPC) (decreasing in $x$ (LPC)) by (P3) of Lemma A1.2, we have $\Delta(x, y) \geq \Delta^{*}(y, z(y))=w\left(z(y), r_{13},-1\right)-w\left(y, r_{23},-1\right)=w\left(z(y), r_{13},-1\right)-($ ay $\mp$ $b z(y)) / c$.

Since $z(y)$ is increasing and $z\left(-r_{23}\right)=-r_{13}, z\left(\left(a-c^{+} r_{23}\right) / b\right)=1$ (UPC), respectively $z(y)$ is decreasing and $z\left(-r_{23}\right)=-r_{13}, z\left(\left(a-c^{-} r_{23}\right) / b\right)=-1$ (LPC), its inverse takes the "“" sign for both (UPC) and (LPC), that is $y=y(z)= \pm\left(a-c^{ \pm} r_{23}\right) / b \cdot z-c^{ \pm} \cdot \sqrt{1-z^{2}}$. Insert it into the function $\Delta^{*}(z)=\Delta^{*}(y(z), z)=w\left(z, r_{23},-1\right)-(a y(z) \mp b z) / c^{ \pm}$to see that $\Delta(x, y) \geq \Delta^{*}$ $(y, z(y))=\Delta^{*}(z)=0$ as desired.

Case 2: Similarly to Case 1, one shows that $z(y)$ is decreasing (UPC) (respectively increasing (LPC)) in the interval $\left(a-c^{ \pm} r_{23}\right) / b \leq y \leq 1$, and in particular $x \geq z(y) \leq z(1)=(a-$ $\left.c^{+} r_{23}\right) / b \geq r_{13}$ (UPC), $x \leq z(y) \leq z(1)=\left(c^{-} r_{23}-a\right) / b \leq r_{13}$ (LPC), where the last inequalities are true because $a-b r_{13}-c^{+} r_{23} \geq c^{+} \cdot\left(1-r_{23}\right) \geq 0$ (UPC), $b r_{13}+a-c^{-} r_{23} \geq c^{-} \cdot\left(1-r_{23}\right) \geq 0$ 
(LPC). Now, we show that $\Delta(x, y)=(a y \mp b x) / c^{ \pm}-w\left(x, r_{13}, 1\right) \geq 0$ under the constraints $z(y) \leq x \leq 1$ (UPC), $-1 \leq x \leq z(y)$ (LPC), $\left(a-c^{ \pm} r_{23}\right) / b \leq y \leq 1$. The function $h(x)=w\left(x, r_{13}, 1\right) \pm$ $b x / c$ is decreasing in the interval $\left(a-c^{+} r_{23}\right) / b \leq x \leq 1$ (UPC), respectively increasing in the interval $-1 \leq x \leq\left(c^{-} r_{23}-a\right) / b$ (LPC):

$$
h^{\prime}(x)= \pm\left(b \pm c r_{13}\right) / c^{ \pm}-a \cdot x / \sqrt{1-x^{2}} \leq(\geq) 0 \quad \Leftrightarrow \quad x \geq\left(a-c^{+} r_{23}\right) / b, \quad x \leq\left(c^{-} r_{23}-a\right) / b
$$

It follows that $\Delta(x, y) \geq \Delta^{*}(y, z(y))=(a y \mp b z(y)) / c^{ \pm}-w\left(z(y), r_{13}, 1\right)$. Since $z(y)$ is decreasing and $z\left(\left(a-c^{+} r_{23}\right) / b\right)=1, z(1)=\left(a-c^{+} r_{23}\right) / b$ (UPC), respectively $z(y)$ is increasing and $z\left(\left(a-c^{-} r_{23}\right) / b\right)=-1, z(1)=\left(c^{-} r_{23}-a\right) / b$ (LPC), its inverse takes the "+" sign for both (UPC) and (LPC), that is $y=y(z)= \pm\left(a-c^{ \pm} r_{23}\right) / b \cdot z+c^{ \pm} \cdot \sqrt{1-z^{2}}$. Insert it into $\Delta^{*}$ $(z)=\Delta^{*}(y(z), z)=(a y(z) \mp b z) / c^{ \pm}-w\left(z, r_{13}, 1\right)$ to see that $\Delta(x, y) \geq \Delta^{*}(y, z(y))=\Delta^{*}(z)=0$.

(3) $(x, t) \in E\left(r_{13}\right)$ and $(y, t) \in E\left(r_{23}\right)$

The validity of the inequality $\frac{\partial}{\partial t} C_{13}(x, t) \leq \frac{\partial}{\partial t} C_{23}(y, t)$ in the Cases (I.a) and (I.b) follows from (A2.1). In Case (I.c) we have $(a y-b x) / c^{+} \leq-1 \leq t$, hence $\frac{\partial}{\partial t} C_{13}(x, t) \geq \frac{\partial}{\partial t} C_{23}(y, t)$. Similarly, the validity of the inequality $\frac{\partial}{\partial t} C_{13}(x, t)+\frac{\partial}{\partial t} C_{23}(y, t)-\frac{1}{2} \geq 0$ in the Cases (I.a) and (I.b) follows from (A2.2). In Case (I.c) we have $(a y+b x) / c^{-} \leq-1 \leq t$, hence $\frac{\partial}{\partial t} C_{13}$ $(x, t)+\frac{\partial}{\partial t} C_{23}(y, t)-\frac{1}{2} \leq 0$.

(4) $(x, t) \in E^{+}\left(r_{13}\right),(y, t)$ arbitrary

(4.1) $x-r_{13}<0$ (UPC), $x-r_{13} \geq 0$ (LPC)

We first note that $\frac{\partial}{\partial t} C_{13}(x, t)=0$ (UPC), $\frac{\partial}{\partial t} C_{13}(x, t)=\frac{1}{2}(\mathrm{LPC})$ :

(UPC): (i) using $x-r_{13}<0: t \leq 1<1+r_{13}-x \Rightarrow(x, t) \notin L_{1}\left(r_{13}\right)$

(ii) using (P5) of Lemma A1.2: $t \geq w\left(x, r_{13}, 1\right)>x+r_{13}-1 \Rightarrow(x, t) \notin L_{4}\left(r_{13}\right)$

(LPC): (P2) of Lemma A1.2: $t \geq w\left(x, r_{13}, 1\right) \geq 1+r_{13}-x \Rightarrow(x, t) \in L_{1}\left(r_{13}\right) \Rightarrow$

We must have $y-r_{23}<0$ in Cases (I.b) and (I.c). We argue as follows:

(UPC) Case (I.b): $x<r_{13} \Rightarrow y<(c+b x) / a \leq\left(c+b r_{13}\right) / a=r_{23}$

(UPC) Case (I.c): if $y \geq r_{23},-x>-r_{13}$ then $a y-b x \geq a r_{23}-b r_{13}=c$ in contradiction to the assumption $a y-b x \leq-c$ defining Case (I.c).

(LPC) Case (I.b): $-x \leq-r_{13} \Rightarrow y<\left(c^{-}-b x\right) / a \leq\left(c^{-}-b r_{13}\right) / a=r_{23}$

(LPC) Case (I.c): if $y \geq r_{23}, x \geq r_{13}$ then $a y+b x \geq a r_{23}+b r_{13}=c^{-}$in contradiction to the assumption $a y+b x \leq-c^{-}$defining Case (I.c).

In this situation, if one assumes $t \geq w\left(y, r_{23}, 1\right)$, then $\frac{\partial}{\partial t} C_{23}(y, t)=0$ in virtue of (3.11):

(UPC): (i) using $y-r_{23}<0$ : $t \leq 1<1+r_{23}-y \Rightarrow(y, t) \notin L_{1}\left(r_{23}\right)$

(ii) using (P5) of Lemma 3.2: $t \geq w\left(y, r_{23}, 1\right)>y+r_{23}-1 \Rightarrow(y, t) \notin L_{4}\left(r_{23}\right)$

(LPC): (i) using $y-r_{23}<0: t \leq 1<1+r_{23}-y \Rightarrow(y, t) \notin L_{1}\left(r_{23}\right)$

(ii) using (P5) of Lemma 3.2: $t \geq w\left(y, r_{23}, 1\right)>y+r_{23}-1 \Rightarrow(y, t) \notin L_{4}\left(r_{23}\right)$

We have three main cases:

(a) $c^{ \pm} t \leq a y \mp b x$ (Case (I.a) and possibly Case (I.b)) 
We have necessarily $\frac{\partial}{\partial t} C_{13}(x, t)=0 \leq \frac{\partial}{\partial t} C_{23}(y, t) \quad(\mathrm{UPC}), \quad \frac{\partial}{\partial t} C_{13}(x, t)+\frac{\partial}{\partial t} C_{23}(y, t)-\frac{1}{2} \geq 0$ (LPC).

(b) $c^{ \pm} t \geq a y \mp b x>c^{ \pm} \cdot w\left(y, r_{23}, 1\right)$ (possible in Cases (I.b) and (I.c))

By the above we have $\frac{\partial}{\partial t} C_{13}(x, t)=\frac{\partial}{\partial t} C_{23}(y, t)=0$ (UPC), $\frac{\partial}{\partial t} C_{13}(x, t)+\frac{\partial}{\partial t} C_{23}(y, t)-\frac{1}{2}=0$ (LPC), which is consistent with the desired inequalities

(c) $c^{ \pm} t \geq a y \mp b x$ and $a y \mp b x \leq c^{ \pm} \cdot w\left(y, r_{23}, 1\right)$ (possible in Cases (I.b) and (I.c))

We derive the inequality (C4.1) $t \geq w\left(x, r_{13}, 1\right) \geq w\left(y, r_{23}, 1\right)$, which again implies that $\frac{\partial}{\partial t}$ $C_{13}(x, t)=\frac{\partial}{\partial t} C_{23}(y, t)=0 \quad(\mathrm{UPC}), \quad \frac{\partial}{\partial t} C_{13}(x, t)+\frac{\partial}{\partial t} C_{23}(y, t)-\frac{1}{2}=0 \quad$ (LPC). We use the transformation $z=z(y)= \pm\left(a y-c^{ \pm} \cdot w\left(y, r_{23}, 1\right)\right) / b$ and its inverse $y=y(z)= \pm\left(a-c^{ \pm} r_{23}\right) /$ $b \cdot z \pm c^{ \pm} \cdot \sqrt{1-z^{2}}$, where the second " \pm " signs hold for both (UPC) and (LPC). We show that $\Delta(x, y)=w\left(x, r_{13}, 1\right)-w\left(y, r_{23}, 1\right) \geq 0$ under the constraints $z(y) \leq x<r_{13}$ (UPC), $r_{13} \leq$ $x \leq z(y)$ (LPC), $-1 \leq y<r_{23}$. By (P4) of Lemma A1.2 the function $w\left(x, r_{13}, 1\right)$ is increasing in $x$ (UPC), respectively decreasing in $x$ (LPC). It follows that $\Delta(x, y) \geq \Delta^{* *}(y, z(y))=w(z(y)$, $\left.r_{13}, 1\right)-w\left(y, r_{23}, 1\right)=w\left(z(y), r_{13}, 1\right)-(a y \mp b z(y)) / c^{ \pm}$.

Insert the inverse $y=y(z)$ into $\Delta^{*}(z)=\Delta^{*}(y(z), z)=w\left(z, r_{13}, 1\right)-(a y(z) \mp b z) / c^{ \pm}$to see that $\Delta(x, y) \geq \Delta^{*}(y, z(y))=\Delta^{*}(z)=a \sqrt{1-z^{2}}(1 \mp 1) \geq 0$ as desired.

(4.2) $x-r_{13} \geq 0$ (UPC), $x-r_{13}<0$ (LPC)

We first note that $\frac{\partial}{\partial t} C_{13}(x, t)=\frac{1}{2}$ (UPC), $\frac{\partial}{\partial t} C_{13}(x, t)=0$ (LPC):

(UPC): Property (P2) implies $t \geq w\left(x, r_{13}, 1\right) \geq 1+r_{13}-x \Rightarrow(x, t) \in L_{1}\left(r_{13}\right)$

(LPC): (i) using $x-r_{13}<0: t \leq 1<1+r_{13}-x \Rightarrow(x, t) \notin L_{1}\left(r_{13}\right)$

(ii) using (P5) of Lemma 3.2: $t \geq w\left(x, r_{13}, 1\right)>x+r_{13}-1 \Rightarrow(x, t) \notin L_{4}\left(r_{13}\right)$

We distinguish between two main cases:

(a) $c^{ \pm} t \geq a y \mp b x$ (Case (I.c) and possibly Case (I.b))

We have necessarily $\frac{\partial}{\partial t} C_{13}(x, t)=\frac{1}{2} \geq \frac{\partial}{\partial t} C_{23}(y, t)$ (UPC), $\frac{\partial}{\partial t} C_{13}(x, t)+\frac{\partial}{\partial t} C_{23}(y, t)-\frac{1}{2} \leq 0$ (LPC)

(b) $a y \mp b x \geq c^{ \pm} t \geq c^{ \pm} \cdot w\left(x, r_{13}, 1\right)$ (Case (I.a) and possibly Case (I.b))

We show that,$\frac{\partial}{\partial t} C_{23}(y, t)=\frac{1}{2}$ which is consistent with the desired inequalities.

We show Case 1: $x \leq\left(b+c^{+} r_{13}\right) / a$ (UPC), $x \geq\left(c^{-} r_{13}-b\right) / a$ (LPC) (C4.2.1) $t \geq w\left(x, r_{13}, 1\right) \geq w$ $\left(y, r_{23}, 1\right)$, and $y-r_{23} \geq 0$ (UPC), $y-r_{23}>0$ (LPC)

Case 2: $x \geq\left(b+c^{+} r_{13}\right) / a(\mathrm{UPC}), x \leq\left(c^{-} r_{13}-b\right) / a(\mathrm{LPC})(\mathrm{C} 4.2 .2) c^{ \pm} \cdot w\left(x, r_{13}, 1\right) \leq c^{ \pm} t \leq$ $a y \mp b x<c^{ \pm} \cdot w\left(y, r_{23},-1\right)$, and $y+r_{23} \geq 0$

Indeed, if these conditions hold, then we have $\frac{\partial}{\partial t} C_{23}(y, t)=\frac{1}{2}$ in virtue of (3.11) as follows:

Case 1: (P2) $\Rightarrow t \geq w\left(y, r_{23}, 1\right) \geq 1+r_{23}-y \Rightarrow(y, t) \in L_{1}\left(r_{23}\right)$

Case 2: $(\mathrm{P} 1) \Rightarrow t<w\left(y, r_{23},-1\right) \leq y+r_{23}-1 \Rightarrow(y, t) \in L_{4}\left(r_{23}\right)$

To show the above inequalities we use the transformation $z=z(x)=\left(c^{ \pm} \cdot w\left(x, r_{13}, 1\right) \pm b x\right) / a$.

Case 1: The function $z(x)$ is increasing in the interval $r_{13} \leq x \leq\left(b+c^{+} r_{13}\right) / a$ (UPC), respectively decreasing in the interval $\left(c^{-} r_{13}-b\right) / a \leq x<r_{13}$ (UPC): $z^{\prime}(x)= \pm\left(b \pm c^{ \pm} r_{13}\right) /$ $a-c^{ \pm} \cdot x / \sqrt{1-x^{2}} \geq(\leq) 0 \quad \Leftrightarrow \quad x \leq\left(b+c^{+} r_{13}\right) / a, x \geq\left(c^{-} r_{13}-b\right) / a$.

In particular $y \geq z(x)>z\left(r_{13}\right)=\left(c^{+}+b r_{13}\right) / a=r_{23}$ (UPC), $y \geq z(x)>z\left(r_{13}\right)=\left(c^{-}-b r_{13}\right) / a=$ $r_{23}$ (LPC). Now, we show that $\Delta(x, y)=w\left(x, r_{13}, 1\right)-w\left(y, r_{23}, 1\right) \geq 0$ under the constraints $z(x) \leq y \leq 1, r_{13} \leq x \leq\left(b+c^{+} r_{13}\right) / a$ (UPC), $\left(c^{-} r_{13}-b\right) / a \leq x<r_{13}$ (LPC). Since $w\left(y, r_{23}, 1\right)$ is 
decreasing in $y$ by (P4) of Lemma 3.2, we have $\Delta(x, y) \geq \Delta^{*}(x, z(x))=w\left(x, r_{13}, 1\right)-w(z(x)$, $\left.r_{23}, 1\right)=(a z(x) \mp b x) / c^{ \pm}-w\left(z(x), r_{23}, 1\right)$.

Since $z(x)$ is increasing and $z\left(r_{13}\right)=r_{23}, z\left(\left(b+c^{+} r_{13}\right) / a\right)=1$ (UPC), respectively $z(x)$ is decreasing, $z\left(r_{13}\right)=r_{23}, z\left(\left(c^{-} r_{13}-b\right) / a\right)=1$ its inverse in (A1.3) takes the "-" sign (UPC), respectively the "+" sign (LPC), that is $x=x(z)= \pm\left(b \pm c^{ \pm} r_{13}\right) / a \cdot z \mp c \cdot \sqrt{1-z^{2}}$. Insert it into $\Delta^{*}(z)=\Delta^{*}(x(z), z)=(a z \mp b x(z)) / c^{ \pm}-w\left(z, r_{23}, 1\right)$ to see that $\Delta(x, y) \geq \Delta^{*}(x, z(x))=\Delta^{*}$ $(z)=0$ as desired.

Case 2: Similarly to Case 1 , one shows that $z(x)$ is decreasing in the interval $\left(b+c^{+} r_{13}\right) /$ $a \leq x \leq 1$ (UPC), respectively $z(x)$ is increasing in the interval $-1 \leq x \leq\left(c^{-} r_{13}-b\right) / a$ (LPC). In particular $y \geq z(x) \geq z(1)=\left(b+c^{+} r_{13}\right) / a \geq-r_{23}$ (UPC), $y \geq z(x) \geq z(-1)=\left(b-c^{-} r_{13}\right) / a \geq-$ $r_{23}$ (LPC), where the last inequalities are true because $a r_{23}+b+c^{+} r_{13}=\left(b+c^{+}\right) \cdot(1+$ $\left.r_{13}\right) \geq 0$ (UPC), $a r_{23}+b-c^{-} r_{13} \geq c^{-} \cdot\left(1-r_{13}\right) \geq 0$ (LPC).

Now, we show that $\Delta(x, y)=w\left(y, r_{23},-1\right)-(a y \mp b x) / c^{ \pm} \geq 0$ under the constraints $z$ $(x) \leq y \leq 1,\left(b+c^{+} r_{13}\right) / a \leq x \leq 1$ (UPC), $-1 \leq x \leq\left(c^{-} r_{13}-a\right) / b$ (LPC). The function $h(y)=$ $w\left(y, r_{23},-1\right)-a y / c^{ \pm}$is increasing in the interval $\left(b \pm c^{ \pm} r_{13}\right) / a \leq y \leq 1$ :

$$
h^{\prime}(y)=\left(c^{ \pm} r_{23}-a\right) / c^{ \pm}+b \cdot y / \sqrt{1-y^{2}} \geq 0 \quad \Leftrightarrow \quad y \geq\left(a-c^{ \pm} r_{23}\right) / b=\left(b \pm c^{ \pm} r_{13}\right) / a
$$

It follows that $\Delta(x, y) \geq \Delta^{*}(x, z(x))=w\left(z(x), r_{23},-1\right)-(a z(x) \mp b x) / c^{ \pm}$. Since $z(x)$ is decreasing and $z\left(\left(b+c^{+} r_{13}\right) / a\right)=1, z(1)=\left(b+c^{+} r_{13}\right) / a$ (UPC), respectively $z(x)$ is increasing and $z$ $\left(\left(c^{-} r_{13}-b\right) / a\right)=1, z(-1)=\left(a-c^{-} r_{23}\right) / b$ (LPC), its inverse takes the "+" sign (UPC), respectively the "-" sign (LPC), that is $x=x(z)= \pm\left(b \pm c^{ \pm} r_{13}\right) / a \cdot z \pm c^{ \pm} \cdot \sqrt{1-z^{2}}$. Insert it into $\Delta^{*}$ $(z)=\Delta^{*}(x(z), z)=w\left(z, r_{23}, 1\right)-(a z \mp b x(z)) / c^{ \pm}$to see that $\Delta(x, y) \geq \Delta^{*}(x, z(x))=\Delta^{*}(z)=0$.

(5) $(y, t) \in E^{+}\left(r_{23}\right),(x, t)$ arbitrary

(5.1) $y-r_{23} \geq 0$

Property (P2) implies $t \geq w\left(y, r_{23}, 1\right) \geq 1+r_{23}-y \Rightarrow(y, t) \in L_{1}\left(r_{23}\right) \Rightarrow \frac{\partial}{\partial t} C_{23}(y, t)=\frac{1}{2}$

We must have $x-r_{13} \geq 0$ (UPC), $x-r_{13} \geq 0$ (LPC), in Cases (I.b) and (I.c):

(UPC) Case (I.b): $x>\left(a y-c^{+}\right) / b \leq\left(a r_{23}-c^{+}\right) / b=r_{13}$

(UPC) Case (I.c): if $-x>-r_{13}, y \geq r_{23}$ then $a y-b x>a r_{23}-b r_{13}=c^{+}$in contradiction to the assumption $a y-b x \leq-c^{+}$defining Case (I.c).

(LPC) Case (I.b): $-y \leq-r_{23} \Rightarrow x<\left(c^{-}-a y\right) / b \leq\left(c^{-}-a r_{23}\right) / b=r_{13}$

(LPC) Case (I.c): if $x \geq r_{13}, y \geq r_{23}$ then $a y+b x \geq a r_{23}+b r_{13}=c^{-}$in contradiction to the assumption $a y+b x \leq-c^{-}$defining Case (I.c).

In this situation, if $t \geq w\left(x, r_{13}, 1\right)$, then $\frac{\partial}{\partial t} C_{13}(x, t)=\frac{1}{2}$ (UPC), $\frac{\partial}{\partial t} C_{13}(x, t)=0$ (LPC):

(UPC): using (P2) of Lemma A1.2: $t \geq w\left(x, r_{13}, 1\right) \geq 1+r_{13}-x \Rightarrow(x, t) \in L_{1}\left(r_{13}\right)$

(LPC):

(i) using $x-r_{13}<0$ : $t \leq 1<1+r_{13}-x \Rightarrow(x, t) \notin L_{1}\left(r_{13}\right)$

(ii) using (P5) of Lemma A1.2: $t \geq w\left(x, r_{13}, 1\right)>x+r_{13}-1 \Rightarrow(x, t) \notin L_{4}\left(r_{13}\right)$

We distinguish between three main cases:

(a) $c^{ \pm} t \leq a y \mp b x$ (Case (I.a) and possibly Case (I.b)) 
We have necessarily $\frac{\partial}{\partial t} C_{13}(x, t) \leq \frac{\partial}{\partial t} C_{23}(y, t)=\frac{1}{2}$ (UPC), $\frac{\partial}{\partial t} C_{13}(x, t)+\frac{\partial}{\partial t} C_{23}(y, t)-\frac{1}{2} \geq 0$ (LPC)

(b) $c^{ \pm} t \geq a y \mp b x>c^{ \pm} \cdot w\left(x, r_{13}, 1\right)$ (possible in Cases (I.b) and (I.c))

By the above we have $\frac{\partial}{\partial t} C_{13}(x, t)=\frac{\partial}{\partial t} C_{23}(y, t)=\frac{1}{2}(\mathrm{UPC}), \frac{\partial}{\partial t} C_{13}(x, t)+\frac{\partial}{\partial t} C_{23}(y, t)-\frac{1}{2}=0$ (LPC), which is consistent with the desired inequalities

(c) $c^{ \pm} t \geq a y \mp b x$ and $a y \mp b x \leq c^{ \pm} \cdot w\left(x, r_{13}, 1\right)$ (possible in Cases (I.b) and (I.c))

We derive the inequality (C5.1) $t \geq w\left(y, r_{23}, 1\right) \geq w\left(x, r_{13}, 1\right)$, which again implies $\frac{\partial}{\partial t} C_{13}$ $(x, t)=\frac{\partial}{\partial t} C_{23}(y, t)=\frac{1}{2}$ (UPC), $\frac{\partial}{\partial t} C_{13}(x, t)+\frac{\partial}{\partial t} C_{23}(y, t)-\frac{1}{2}=0$ (LPC). We use the transformation $z=z(x)=\left(c \cdot w\left(x, r_{13}, 1\right) \pm b x\right) / a$ and its inverse in (A1.3) to show that $\Delta(x, y)=$ $w\left(y, r_{23}, 1\right)-w\left(x, r_{13}, 1\right) \geq 0$ under the constraints $r_{23} \leq y \leq z(x), r_{13} \leq x \leq 1$ (UPC), $-1 \leq x<$ $r_{13}$ (LPC). By (P4) of Lemma A1.2 the function $w\left(y, r_{23}, 1\right)$ is decreasing in $y$, hence $\Delta(x, y) \geq \Delta^{*}(x, z(x))=w\left(z(x), r_{23}, 1\right)-w\left(x, r_{13}, 1\right)=w\left(z(x), r_{23}, 1\right)-(a z(x) \div b x) / c^{ \pm}$.

Insert the inverse $x=x(z)$ into $\Delta^{*}(z)=\Delta^{*}(x(z), z)=w\left(z, r_{23}, 1\right)-(a z \mp b x(z)) / c^{ \pm}$to see that $\Delta(x, y) \geq \Delta^{*}(x, z(x))=\Delta^{*}(z)=b \sqrt{1-z^{2}}(1 \pm 1) \geq 0$ as desired.

(5.2) $y-r_{23}<0$

We first note that $\frac{\partial}{\partial t} C_{23}(y, t)=0$ :

(i) using $y-r_{23}<0$ : $t \leq 1<1+r_{23}-y \Rightarrow(y, t) \notin L_{1}\left(r_{23}\right)$

(ii) using (P5) of Lemma 3.2: $t \geq w\left(y, r_{23}, 1\right)>y+r_{23}-1 \Rightarrow(y, t) \notin L_{4}\left(r_{23}\right)$

We distinguish between two main cases:

(a) $c^{ \pm} t \geq a y \mp b x$ (Case (I.c) and possibly Case (I.b))

We have necessarily $\frac{\partial}{\partial t} C_{13}(x, t) \geq \frac{\partial}{\partial t} C_{23}(y, t)=0 \quad$ (UPC), $\frac{\partial}{\partial t} C_{13}(x, t)+\frac{\partial}{\partial t} C_{23}(y, t)-\frac{1}{2} \leq 0$ (LPC)

(b) $c^{ \pm} \cdot w\left(y, r_{23}, 1\right) \leq c^{ \pm} t \leq a y \mp b x$ (Case (I.a) and possibly Case (I.b))

We show that $\frac{\partial}{\partial t} C_{13}(x, t)=0$ (UPC), $\frac{\partial}{\partial t} C_{13}(x, t)=\frac{1}{2}$ (LPC), which is consistent with the desired inequalities $\frac{\partial}{\partial t} C_{13}(x, t) \leq \frac{\partial}{\partial t} C_{23}(y, t)=0$ (UPC), $\frac{\partial}{\partial t} C_{13}(x, t)+\frac{\partial}{\partial t} C_{23}(y, t)-\frac{1}{2} \geq 0$ (LPC)

Case $1 \quad y \geq\left(c^{ \pm} r_{23}-a\right) / b$

(C5.2.1) $t \geq w\left(y, r_{23}, 1\right) \geq w\left(x, r_{13}, 1\right)$, and $x-r_{13}<0$ (UPC), $x-r_{13}>0$ (LPC)

Case $2 \quad y \leq\left(c^{ \pm} r_{23}-a\right) / b$

(C5.2.2) $c^{ \pm} \cdot w\left(y, r_{23}, 1\right) \leq c^{ \pm} t \leq a y \mp b x<c^{ \pm} \cdot w\left(x, r_{13},-1\right), x+r_{13}<0$ (UPC), $x+r_{13} \geq$ 0 (LPC)

Indeed, if these conditions hold, then $\frac{\partial}{\partial t} C_{13}(x, t)=0$ in virtue of (3.11) as follows:

UPC) Case 1 (i) using $x-r_{13}<0$ : $t \leq 1<1+r_{13}-x \Rightarrow(x, t) \notin L_{1}\left(r_{13}\right)$

(ii) using (P5) of Lemma 3.2: $t \geq w\left(x, r_{13}, 1\right)>x+r_{13}-1 \Rightarrow(x, t) \notin L_{4}\left(r_{13}\right)$

(LPC) Case 1: (P2) $\Rightarrow t \geq w\left(x, r_{13}, 1\right) \geq 1+r_{13}-x \Rightarrow(x, t) \in L_{1}\left(r_{13}\right)$

(UPC) Case 2: (i) using (P6) of Lemma 3.2: $t \leq w\left(x, r_{13},-1\right)<1+r_{13}-x \Rightarrow(x, t) \notin L_{1}\left(r_{13}\right)$

(ii) using $x+r_{13}<0 t \geq-1>x+r_{13}-1 \Rightarrow(x, t) \notin L_{4}\left(r_{13}\right)$

(LPC) Case 2: (P1) $\Rightarrow t<w\left(x, r_{13},-1\right) \leq x+r_{13}-1 \Rightarrow(x, t) \in L_{4}\left(r_{13}\right)$ 
To show the above inequalities we use the transformation $z=z(y)= \pm\left(a y-c^{ \pm} \cdot w(y\right.$, $\left.\left.r_{23}, 1\right)\right) / b$.

Case 1: Since the function $z(y)$ is increasing (UPC) (respectively decreasing (LPC)) in the interval $\left(c^{ \pm} r_{23}-a\right) / b \leq y<r_{23}$, we have in particular $x \leq z(y)<z\left(r_{23}\right)=\left(a r_{23}-c^{+}\right) / b=$ $r_{13}$ (UPC), $x \geq z(y)>z\left(r_{23}\right)=\left(c^{-}-a r_{23}\right) / b=r_{13}$ (LPC). We show that $\Delta(x, y)=w\left(y, r_{23}, 1\right)-$ $w\left(x, r_{13}, 1\right) \geq 0$ under the constraints $-1 \leq x \leq z(y)$ (UPC), $z(y) \leq x \leq 1$ (LPC), $\left(c^{ \pm} r_{23}-a\right) / b \leq$ $y<r_{23}$. With (P4) of Lemma A1.2, $w\left(x, r_{13}, 1\right)$ is increasing in $x$ (UPC), decreasing in $x$ (LPC), hence $\Delta(x, y) \geq \Delta^{*}(y, z(y))=w\left(y, r_{23}, 1\right)-w\left(z(y), r_{13}, 1\right)=(a y \mp b z(y)) / c^{ \pm}-w\left(z(y), r_{13}, 1\right)$.

Since $z(y)$ is increasing and $z\left(r_{23}\right)=r_{13}, z\left(\left(c^{+} r_{23}-a\right) / b\right)=-1$ (UPC), respectively $z(y)$ is decreasing and $z\left(r_{23}\right)=r_{13}, z\left(\left(c^{-} r_{23}-a\right) / b\right)=1$ (LPC), its inverse in (A1.3) takes the "+" sign: $y(z)= \pm\left(a-c r_{23}\right) / b \cdot z+c \sqrt{1-z^{2}}$. Insert into $\Delta^{*}(z)=\Delta^{*}(y(z), z)=(a y(z) \mp b z) / c^{ \pm}-$ $w\left(z, r_{23}, 1\right)$ to see that $\Delta(x, y) \geq \Delta^{*}(y, z(y))=\Delta^{*}(z)=0$ as desired.

Case 2: One shows that $z(y)$ is decreasing (UPC) (respectively increasing (LPC)) in the interval $-1 \leq y \leq\left(c^{ \pm} r_{23}-a\right) / b$. In particular $x \leq z(y) \leq z(-1)=\left(c^{+} r_{23}-a\right) / b \leq-r_{13}$ (UPC), $x \geq z(y) \geq z(-1)=\left(a-c^{-} r_{23}\right) / b \geq-r_{13}$ (LPC), where the last inequalities are true because $a-b r_{13}-c^{+} r_{23} \geq c^{+} \cdot\left(1-r_{23}\right) \geq 0$ (UPC), $a+b r_{13}-c^{-} r_{23} \geq c^{-} \cdot\left(1-r_{23}\right) \geq 0$ (LPC).

Now, we show that $\Delta(x, y)=w\left(x, r_{13},-1\right)-(a y \mp b x) / c^{ \pm} \geq 0$ under the constraints $1 \leq x \leq z(y)$ (UPC), $z(y) \leq x \leq 1$ (LPC), $-1 \leq y \leq\left(c^{ \pm} r_{23}-a\right) / b$. Since the function $h(x)=w$ $\left(x, r_{13},-1\right) \pm b x / c$ is decreasing (UPC), respectively increasing (LPC), we have $\Delta(x$, $y) \geq \Delta^{*}(y, z(y))=w\left(z(y), r_{13},-1\right)-(a y \mp b z(y)) / c^{ \pm}$. Since $z(y)$ is decreasing and $z\left(\left(c^{+} r_{23}-\right.\right.$ $a) / b)=-1, z(-1)=\left(c^{+} r_{23}-a\right) / b$ (UPC), respectively $z(y)$ is increasing and $z\left(\left(c^{-} r_{23}-a\right) /\right.$ $b)=1, \quad z(-1)=\left(a-c^{-} r_{23}\right) / b$ (LPC), its inverse in (A1.3) takes the "-" sign, that is $y(z)= \pm\left(a-c^{ \pm} r_{23}\right) / b \cdot z-c^{ \pm} \cdot \sqrt{1-z^{2}}$. Insert it into $\Delta^{*}(z)=\Delta^{*}(y(z), z)=w\left(z, r_{13},-1\right)-(a y$ $(z)-b z) / c$ to see that $\Delta(x, y) \geq \Delta^{* *}(y, z(y))=\Delta^{* *}(z)=0$ as desired.

\section{Authors information}

URL: http://sites.google.com/site/whurlimann/

\section{Acknowledgement}

The author is grateful to the referees for their useful comments, which led to some improvement in content.

Received: 4 April 2013 Accepted: 27 June 2013

Published: 11 June 2014

\section{References}

Allen, DE, Ashraf, MA, McAleer, M, Powell, RJ, Singh, AK: Financial dependence analysis: applications of vine copulae. (2013). http://eprints.ucm.es/17819/1/1305.pdf Accessed 31 May 2013

Bedford, T, Cooke, R: Probability density decomposition for conditionally dependent random variables modelled by vines. Ann. Math. Artif. Intell. 32, 245-268 (2001)

Bedford, T, Cooke, R: Vines - a new graphical model for dependent random variables. Ann. Statist. 30(4), $1031-1068(2002)$

Bernard, C, Czado, C: Multivariate Option Pricing Using Copulae. Appl. Stoch. Models Bus. Ind. (2012). doi:10.1002/ asmb.1934

Brechmann, EC, Czado, C: Risk management with high-dimensional vine copulas: An analysis of the Euro Stoxx 50. Statist. Risk Modeling (2013) in press

Carathéodory, C: Über den Variabilitätsbereich der Fourierschen Konstanten von positiven harmonischen Funktionen. Rend. del Circolo Matem. di Palermo 32, 193-217 (1911)

Cherubini, U, Luciano, E, Vecchiato, W: Copula Methods in Finance. J. Wiley Finance Series, New York (2004)

Czado, C: Pair-copula construction of multivariate copulas. In: Jaworski, P., Durante, F., Härdle, W., Rychlik, T. (eds.) Copula Theory and its Applications. Proc. Workshop held in Warsaw, 25-26 Sep. 2009. Lecture Notes in Statistics, vol. 198, pp. 93-109. Springer, Heidelberg (2010)

Czado, C, Schepsmeier, U, Min, A: Maximum likelihood estimation of mixed C-vines with application to exchange rates. Statist. Modelling 12(3), 229-255 (2012)

Devroye, L, Letac, G: Copulas in three dimensions with prescribed correlations. (2010). http://arxiv.org/abs/1004.3146v1 [math.ST] Accessed 31 May 2013

Durante, F, Klement, EP, Quesada-Molina, JJ: Copulas: compatibility and Fréchet classes. (2007a). http://arxiv.org/abs/ 0711.2409v1 [math.ST] (2007a). Accessed 31 May 2013 
Durante, F, Klement, EP, Quesada-Molina, JJ, Sarkoci, P: Remarks on two product-like constructions for copulas. Kybernetika 43(2), 235-244 (2007b)

Ghosh, S: Dependence in stochastic simulation models. Cornell University, Dissertation (2004). http://www.research.ibm. com/people/g/ghoshs/pubs/thesis.pdf. Accessed 31 May 2013

Ghosh, S, Henderson, SG: Properties of the NORTA method in higher dimensions. In: Proceedings of the 2002 Winter Simulation Conference, Sch. of Operations Res. \& Ind. Eng.,Cornell Univ., Ithaca, NY, USA, pp. 263-269. (2002)

Hürlimann, W: Random loss development factor curves and stochastic claims reserving. JP J Fund Appl Stat 1(1), 49-62 (2011)

Hürlimann, W: Compatibility conditions for the multivariate normal copula with given rank correlation matrix. Pioneer $J$ Theor Appl Stat 3(2), 71-86 (2012a)

Hürlimann, W: Positive semi-definite correlation matrices: recursive algorithmic generation and volume measure. Pure Math Sci 1(3), 137-149 (2012b)

Hürlimann, W: On trivariate copulas with bivariate linear Spearman marginal copulas. J. Math. Syst Sci 2 $368-383(2012 c)$

Joe, $\mathrm{H}$ : Families of $\mathrm{m}$-variate distributions with given margins and $\mathrm{m}(\mathrm{m}-1) / 2$ bivariate dependence parameters. In: Rüschendorf, L., Schweizer, B, Taylor, MD (eds.) Distributions with Fixed Marginals and Related Topics. IMS Lecture Notes Monograph Series 28, pp. 120-141. Hayward, CA (1996)

Joe, H: Multivariate models and dependence concepts. In: Monographs on Statistics and Applied Probability, vol. 73. Chapman \& Hall, London (1997)

Joe, H, Kurowicka, D (eds.): Dependence Modeling - Vine Copula Handbook. World Scientific Publishing Co., Singapore (2011)

Kurowicka, D, Misiewicz, J, Cooke, R, Elliptical copulae: Proceedings of the International Conference on Monte Carlo Simulation, Monte Carlo. In: GI Schuëller \& PD Spanos (Eds.) Proceedings Monte Carlo Simulation, Lisse: Balkema, pp. 209-214. (2000)

Kurowicka, D, Cooke, R: Conditional, partial and rank correlation for the elliptical copula; dependence modelling in uncertainty analysis. TR, Delft Univ Technol. In: E Zio, M Demichela \& N Piccinini (Eds.), Proceedings ESREL 2001, Torino: Politecnico di Torino, pp. 1795-1802.

Kurowicka, D, Cooke, R: Uncertainty Analysis with High Dimensional Dependence Modelling. J. Wiley, Chichester (2006)

Letac, G: Jacobi polynomials and joint distributions in Rn with beta margins and prescribed correlation matrices. Warwick Workshop, Centre Res Stat Methodol (2010). http://www2.warwick.ac.uk/fac/sci/statistics/crism/workshops/ orthogonal-polynomials/speakers/gerard_letac.pdf. Accessed 31 May 2013

Mauguis, P-A, Guégan, D: An econometric study of vine copulas. Int. J. Economics and Finance 2(5), 2-14 (2010)

Perlman, MD, Wellner, JA: Squaring the circle and cubing the sphere: circular and spherical copulas. Symmetry 3, 574-599 (2011)

Sklar, A: Fonctions de répartition et leurs marges. Publications de l'Institut Statistique de l'Université de Paris 8, 229-31 (1959)

Steinitz, E: Bedingt konvergente Reihen und konvexe Systeme. J Reine Angew. Math. 143, 128-175 (1914)

Vaz de Melo Mendes, B, Mendes Semeraro, M, Câmara Leal, RP: Pair-copula modeling in finance. Financial Markets and Portfolio Management 24(2), 193-213 (2010)

Ycart, B: Extreme points in convex sets of symmetric matrices. Proc. Amer. Math. Soc. 95(4), 607-612 (1985)

doi:10.1186/2195-5832-1-7

Cite this article as: Hürlimann: A closed-form universal trivariate pair-copula. Journal of Statistical Distributions and Applications 2014 1:7.

\section{Submit your manuscript to a SpringerOpen ${ }^{\circ}$ journal and benefit from:}

- Convenient online submission

- Rigorous peer review

- Immediate publication on acceptance

- Open access: articles freely available online

- High visibility within the field

- Retaining the copyright to your article

Submit your next manuscript at $\boldsymbol{\nabla}$ springeropen.com 